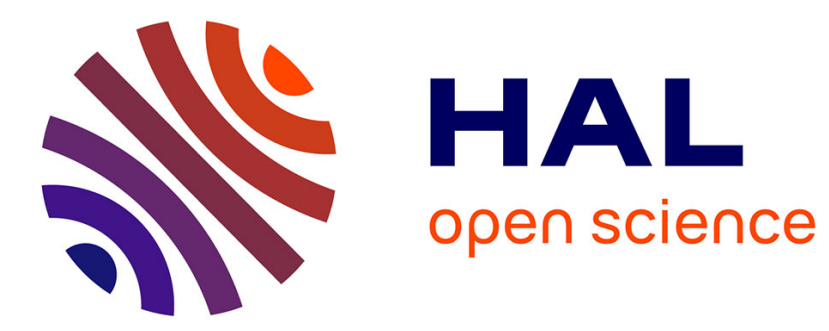

\title{
Polyploidy in liver development, homeostasis and disease
} Romain Donne, Maëva Saroul-Aïnama, Pierre Cordier, Séverine Celton-Morizur, Chantal Desdouets

\section{To cite this version:}

Romain Donne, Maëva Saroul-Aïnama, Pierre Cordier, Séverine Celton-Morizur, Chantal Desdouets. Polyploidy in liver development, homeostasis and disease. Nature reviews. Gastroenterology \& hepatology, 2020, 17 (7), pp.391-405. 10.1038/s41575-020-0284-x . inserm-02992166

\section{HAL Id: inserm-02992166 https://www.hal.inserm.fr/inserm-02992166}

Submitted on 6 Nov 2020

HAL is a multi-disciplinary open access archive for the deposit and dissemination of scientific research documents, whether they are published or not. The documents may come from teaching and research institutions in France or abroad, or from public or private research centers.
L'archive ouverte pluridisciplinaire HAL, est destinée au dépôt et à la diffusion de documents scientifiques de niveau recherche, publiés ou non, émanant des établissements d'enseignement et de recherche français ou étrangers, des laboratoires publics ou privés. 
8

Polyploidy in liver development, homeostasis and disease

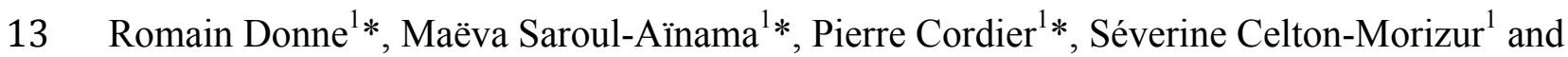
14 Chantal Desdouets**1

15 1- Team Proliferation, Stress and Liver Physiopathology, Centre de Recherche des 16 Cordeliers, INSERM, Sorbonne Université, USPC, Université de Paris, Paris, France. 17

* Equal contribution

**e-mail: chantal.desdouets@inserm.fr, ORCID number : 0000-0002-1955-8915 
1 Abstract | Polyploidy (or whole-genome duplication) is the condition of having more than

2 two basic sets of chromosomes. Polyploidization is well tolerated in many species and can

3 lead to specific biological functions. In mammals, programmed polyploidization takes place

4 during development in certain tissues such as the heart and placenta and is considered to be a

5 feature of differentiation. However, unscheduled polyploidization can cause genomic

6 instability and has been observed in pathological conditions, such as cancer. Polyploidy of the

7 liver parenchyma was first described more than 100 years ago. It's one of the few mammalian

8 organs that display changes in polyploidy during homeostasis, regeneration and in response to

9 damage. In the human liver, around $30 \%$ of hepatocytes are polyploid. The polyploidy of

10 hepatocytes results from both nuclear polyploidy (an increase in the amount of DNA per

11 nucleus) and cellular polyploidy (an increase in the number of nuclei per cell). In this Review,

12 we discuss the regulation of polyploidy in liver development and pathophysiology. We also

13 provide an overview of current knowledge about the mechanisms of hepatocyte

14 polyploidization, its biological importance and the fate of polyploid hepatocytes during liver

15 tumorigenesis.

Author contributions

R.D., M.S-A. and P.C. wrote the article and researched data for the article. S.C.-M. wrote the article and made a substantial contribution to the discussion of content. C.D. wrote the article, researched data for the article, made a substantial contribution to the discussion of content, and reviewed/edited the manuscript before submission.

21 Competing interests

The authors declare no competing interests.

23 Publisher's note

23 springer Nature remains neutral with regard to jurisdictional claims in published maps and institutional affiliations.

24 Peer review information

Nature Reviews XXX thanks [Referee\#1 name], [Referee\#2 name] and the other, anonymous, reviewer(s) for their contribution to the peer review of this work. [Au: inserted this as a placeholder for now] 


\section{Key points}

- Polyploidy, a condition in which cells contain more than two sets of homologous chromosomes, is a well-known feature of mammalian hepatocytes.

- Polyploidy is defined on the basis of the DNA content of each nucleus (nuclear ploidy; for example, $2 n, 4 n$ or $8 n$ ) and the number of nuclei per cell (cellular ploidy).

- The adult liver contains a heterogeneous mixture of diploid and polyploid hepatocytes.

- The liver is one of the few mammalian organs that display changes in ploidy during normal homeostasis, regeneration and in response to damage.

- Polyploid state could provide protection from tumorigenesis by providing extra copies of tumour suppressor copies.

- Amplification of nuclear ploidy within liver tumours is associated with a poor prognosis. 


\section{Introduction}

3

4

The chromosome houses the genetic information specific to all living beings. Each species has a specific number, $n$, of chromosomes in its haploid genome. Thus, Homo sapiens cells have $n=23$ chromosomes and Mus musculus cells have $n=20$ chromosomes in their haploid genome ${ }^{1}$. Eukaryotic organisms typically have two complete sets of homologous chromosomes. However, the number of a set of chromosomes can differ between cells or species. Cells with only one copy of each chromosome $(n)$ are described as haploid, those with two copies are diploid $(2 n)$, three copies are triploid $(3 n)$, four copies are tetraploid $(4 n)$, and so on (FIG. 1A). The presence of more than two complete sets of chromosomes is known as polyploidy or whole-genome duplication ${ }^{2}$. The polyploid state should not be confused with aneuploidy, in which the copy number of whole chromosomes or chromosome segments is modified, either by a gain or a loss ${ }^{3}$. In polyploid species, the additional chromosome sets can have different origins. Allopolyploidy arises through the fusion of two or more cells with distantly related genomes (that is, from different species). Autopolyploidy arises through the duplication of a single genome or the fusion of closely related genomes (that is, from the same species). Finally, the number of chromosome sets can be amplified within a single nucleus (mononucleate polyploid populations), defining the nuclear ploidy, or, as in some polyploid cells, the genetic material can be distributed between two or more nuclei, defining the cellular ploidy (FIG. 1B).

Polyploidy is not rare in eukaryotes, and is now considered a common mode of speciation, with consequences for evolution and biodiversity ${ }^{4-7}$. Indeed, polyploidy is a feature of plant genomes, contributing to variations in both genome size and gene content ${ }^{8-10}$. Polyploidy has been observed in most plant groups, but it is most frequent in angiosperms ${ }^{11-13}$. It has also been reported in some insects, fishes, amphibians and reptiles ${ }^{7,14}$. In mammals, whole- 
1 organism polyploidization is rare, as it typically leads to embryonic resorption or spontaneous

2 abortion ${ }^{15-17}$. However, the red viscacha rat (Tympanoctomys barrerae) and its close

3 relatives, which are fully tetraploid, are the exception to the rule ${ }^{18}$. The emergence of

4 polyploid cells in mammals can be associated with the development and differentiation of

5 certain tissues. For instance, polyploid cells are present in the heart (cardiomyocytes: $4 n$ ),

6 placenta (trophoblast giant cells: $8 n$ to $64 n$ ), bone marrow (megakaryocytes: $16 n$ to $128 n$ ),

7 pancreas (acinar cells: $4 n$ ) ${ }^{19,20}$ and liver (hepatocytes: $4 n$ to $\left.8 n\right)^{21-23}$. Over the past decade,

8 several breakthroughs have occurred in determining the role of polyploidy in regulating organ

9 size, tissue regeneration and repair ${ }^{24,25}$. In Drosophila melanogaster and some vertebrate

10 tissues, polyploidy provides an alternative means of compensating for cell loss, particularly in

11 post-mitotic tissues that lack stem cells ${ }^{25}$. For example, polyploidy has a crucial role in heart

12 and kidney regeneration and repair processes in mammals ${ }^{25-28}$. One major concern is the

13 association of an accumulation of polyploid contingents with many age-related diseases,

14 including arterial hypertension, hyperthyroidism, metabolic disorders and cancer ${ }^{29-33}$.

15 Proliferating polyploid cells have been shown to be genetically unstable, thereby potentially

16 facilitating tumour development ${ }^{21,34}$. In addition, there is growing evidence to suggest that

17 polyploid intermediates play a key part in shaping the composition of cancer genomes: most

18 solid tumours have polyploid or near-polyploid karyotypes ${ }^{35,36}$.

19 Thus, several key questions arise. Does polyploidy confer selective benefits and a protective

20 role in evolution, or does it generate a key contingent of cells that underlie tumorigenesis?

21 This Review focuses on the duality of the polyploid state, and on the mechanisms leading to

22 it. In this Review, we will first introduce the different mechanisms leading to polyploidy.

23 Next, we will examine how polyploid hepatocytes are generated during physiological and

24 pathological situations. Finally, we will discuss the effects of polyploidy on liver function. 


\section{1 [H1] Mechanisms leading to polyploidy}

2

3 How does a diploid cell become polyploid? In a physiological or pathological context, various mechanisms can promote the genesis of polyploid cells: endoreplication, cytokinesis failure and cell fusion, which is not dependent on the cell cycle and division (FIG. 2).

\section{[H2] Cell fusion}

Fusion can occur between cells of the same type (homotypic) or cells of different origins (heterotypic) (FIG. 2A). Cells that fuse without nuclear fusion are described as syncytia. The major cell fusion event that modifies ploidy is fertilization (an example of heterotypic cell fusion) ${ }^{37}$. Cell fusion is also a key process in tissue development and homeostasis. For example, myoblasts fuse to form myotubes in muscle, macrophages fuse to form osteoclasts in bone, and cytotrophoblasts fuse to form placental syncytiotrophoblasts ${ }^{38-40}$. Cell fusion mechanisms have been analysed in detail in several experimental models, in particular in myoblast differentiation ${ }^{41}$. Cell fusion requires cell-cell adhesion via the repulsive charges of the two cells' membranes, followed by destabilization of the opposing lipid bilayers, leading to fusion, pore formation and expansion. Myoblasts use actin-propelled membrane protrusions to promote fusogenic protein engagement and fusion pore formation ${ }^{42}$. Cell fusion also has a key role in wound healing and tissue regeneration. In injured tissues, bone marrow-derived cells can fuse to differentiated cells to form hybrids with regenerative potential ${ }^{43}$. Such heterotypic cell fusion has been observed in various human and mice organs, including the muscle, brain, retina, intestine and liver, and it has been shown to participate in the reestablishment of tissue function ${ }^{44-48}$.

Viral infections can also have an important role in the formation of polyploid cells ${ }^{49}$. The best-documented example is that of human papillomavirus (HPV), the major aetiological agent of cervical cancer ${ }^{50}$. The expression of the viral HPV-16 E5 oncoprotein at the surface of infected cervical epithelial cells is sufficient for the formation of tetraploid binucleate cells 
51,52. Tetraploid cervical cells are observed in premalignant lesions of human cervical cancer and it has been established as a prognostic factor that allows estimating the relative

3 progression risk into more advanced lesion ${ }^{53}$. Interestingly, women diagnosed as low-grade

4 squamous intraepithelial lesion/HPV-positive exhibit elevated levels of tetraploid cervical 5 cells compared with normal/HPV-negative women, indicating that formation of tetraploid 6 cells is obviously associated with HPV infection ${ }^{54}$.

\section{7 [H2] Endoreplication}

8 The canonical cell cycle of mammals has four successive phases: G1, S, G2 and M (FIG. 2B).

9 This process is governed by the cyclin-dependent kinases (CDKs), a family of serine10 threonine protein kinases. CDK activity is dependent on association with non-catalytic 11 regulatory subunits called cyclins. $\mathrm{CDK}$-cyclin complexes phosphorylate specific substrates to induce DNA replication (which is driven by the CDK responsible for S phase entry, S-

$13 \mathrm{CDK}$ ) and mitosis (which is driven by the CDK responsible for M phase entry, M-CDK), the 14 two major events of the cell division cycle ${ }^{55}$. In mammals, the $\mathrm{S}$ and $\mathrm{M}$ phases are controlled by CDK2 associated with cyclins $\mathrm{E}$ and $\mathrm{A}$, and by CDK1 associated with cyclins $\mathrm{B}$ and $\mathrm{A}$, 16 respectively. These two steps are coupled, such that mitosis phase cannot begin until S phase has been completed. Endoreplication is an alternative cell cycle in which cells successively replicate their genomes in the absence of cell division ${ }^{56-59}$. This process leads to the genesis of mononucleate polyploid progenies. There are two variants of the endoreplication process: endocycles and endomitosis (FIG. 2C). During endocycles, cells alternate between G and S 21 phases, whereas, during endomitosis, cells abort mitosis by skipping metaphase, anaphase or 22 cytokinesis ${ }^{60}$. Several elegant reviews have highlighted the mechanisms that regulate endocycles in different species and cell types ${ }^{25,57,61}$. The key event that triggers endocycles

24 is the inhibition of entry into mitosis. This inhibition might be mediated by the proteolysis of 25 mitotic cyclins under the control of an E3 ubiquitin ligase, the APC/C (anaphase-promoting 
1 complex; also known as the cyclosome). M-CDK can also be inhibited by interactions with

2 cyclin kinase inhibitors (CKIs). A second crucial event is the oscillation of S-CDK activity

3 between low and high levels in the $\mathrm{G}$ and $\mathrm{S}$ phases, respectively, which is essential for

4 genome reduplication in the absence of cell division. One of the best-characterized examples

5 of an endocycle in mammals is observed in the trophoblast giant cells. Trophoblast giant cell

6 polyploidization is crucial for implantation and for the modulation of post-implantation 7 placentation ${ }^{62}$. In particular, the accumulation of the CKIs p57 and p21 is essential for 8 endocycle induction, as it enhances cyclin B degradation and inhibits CDK1 activity ${ }^{63,64}$.

9 Also, E2F transcription factors are required for the regulation of trophoblast giant cell 10 polyploidization ${ }^{65,66}$. This point is discussed in more detail later.

11 The observation in different experimental models of a connection between genome instability 12 and endoreplication cycles is a major source of concern ${ }^{57,67}$. For instance, double-strand 13 break induction is common in the leaves of Arabidopsis thaliana plants subjected to UV 14 irradiation. Induction of the DNA damage response results in G2-M arrest. If the DNA 15 damage response persists, endoreplication is triggered ${ }^{68,69}$. In this context, it has been 16 suggested that endoreplication is a strategy used by the plant to sustain growth under 17 genotoxic stress. In human and mouse cell lines, that have high amounts of dysfunctional 18 telomeres, endoreplication is induced by $\mathrm{p} 53 / \mathrm{pRb}{ }^{70,71}$. This endoreplication requires 19 persistent serine-protein kinase ATM and/or ATR (ATM and RAD3-related)-dependent 20 signalling, which is induced by irreparably damaged telomeres. Prolonged cell cycle arrest in 21 G2 leads to mitosis being bypassed, which results in the genesis of mononucleate tetraploid 22 progenies $^{70,71}$.

23 Endomitosis has been linked to problems in metaphase-anaphase transition. Thus, cancer 24 cells treated with antimitotic drugs that target microtubule dynamics (for example, taxanes, 25 vinca alkaloids and epothilones) are blocked in metaphase owing to spindle assembly 
1 checkpoint (SAC) activation ${ }^{72}$. The SAC prevents passage through anaphase until all

2 chromosomes are properly attached to kinetochores ${ }^{73}$. Sustained SAC activation results in

3 either cell death through a phenomenon called mitotic catastrophe (leading to cancer

4 remission), or mitosis exit in metaphase, also known as mitotic slippage, driving cancer

5 progression ${ }^{74-76}$. The outcome is dictated by the balance between the opposing activities of

6 the apoptotic machinery and two mitotic ubiquitin ligases. Slippage is thought to involve the 7 gradual proteolysis of cyclin B1 by the APC/C and CRL2 ${ }^{\mathrm{ZYG11A} / \mathrm{B}}$ ubiquitin ligases ${ }^{77-79}$. How

8 cancer cells treated with spindle poisons (e.g. taxol) avoid apoptosis during mitosis remains

9 unclear. It has been suggested that polyploidy might confer a survival advantage on tumour 10 cells, contributing to the evolution of treatment resistance ${ }^{80}$. Mitotic slippage events have 11 also been described during colorectal cancer cell divisions in tumours with adenomatous 12 polyposis coli $(A P C)$ gene mutations ${ }^{81}$. APC mutations are the most frequent type of mutation 13 in human colorectal tumours ${ }^{82}$. APC is involved in the WNT signalling pathway, but it also 14 binds to mitotic spindle microtubules, and its absence impairs kinetochore-microtubule 15 interactions, leading to chromosomal instability (CIN) in APC-driven murine colon 16 carcinomas ${ }^{83}$. Dikovskaya and co-workers have demonstrated that conditional knockout of $17 A P C$ in vitro and in vivo induces mitotic slippage that is linked to the genesis of a 18 mononucleate tetraploid cell contingent ${ }^{81}$. However, the link between tetraploid cells with $19 A P C$ mutations and the increase in $\mathrm{CIN}$ in colon carcinogenesis remains unclear.

\section{0 [H2] Cytokinesis failure}

21 Cytokinesis is the final step in cell division and is initiated during anaphase ${ }^{84}$. It involves a 22 finely regulated series of events to ensure that genomic and cytoplasmic materials are evenly 23 distributed between the two nascent daughter cells. Four events must occur for the correct 24 induction of cytokinesis: establishment of the division plane, contraction of the actomyosin 25 ring, ingression of the cleavage furrow and, finally, formation of the intracellular bridge, the 
1 degradation of which leads to cell abscission. Interference with the progression of these steps

2 can lead to cytokinesis failure and to the genesis of binucleate polyploid cells (FIG. 2C) ${ }^{85,86}$.

3 Cytokinesis failure has been known for several decades to be a physiological process involved

4 in the development of certain human tissues and organs, such as the heart ${ }^{87}$, bone marrow ${ }^{88}$

5 and liver ${ }^{89}$. For example, ventricular cardiomyocytes respond to the increase in blood flow

6 after birth with an adaptive increase in volume (known as hypertrophy) ${ }^{90}$. This transition from

7 hyperplasia to hypertrophy is clearly associated with polyploidization as it leads to the genesis

8 of binucleate tetraploid cardiomyocytes ${ }^{91}$. The degree of polyploidization in ventricular

9 cardiomyocytes differs between species and varies with age, but it might reach $85 \%$ in 10 rodents and 30\% in humans $91,92,93,94$. Cyclin G1 has been identified as an important player 11 in the molecular machinery that controls cardiomyocyte binucleation. The expression of 12 cyclin G1 in neonatal cardiomyocytes promotes G1-S cell cycle transition but inhibits 13 cytokinesis ${ }^{95}$. Cytokinesis failure in cardiomyocytes might also be caused by the abnormal 14 localization of proteins that regulate the organization of the contractile ring (for example, Ras 15 homolog gene family member A (RhoA), ROCK-I, ROCK-II and anillin) ${ }^{87,96,97 .}$

16 Cytokinesis failure has also been reported in a number of diseases, often in association with 17 high rates of mutation or genomic instability ${ }^{98-100}$. In their elegant review, Lacroix and 18 Maddox describe several examples of cytokinesis failure events in different diseases: 19 Wiskott-Aldrich syndrome, X-linked neutropenia, Fanconi anaemia, Lowe syndrome, type II 20 neurofibromatosis and age-related macular degeneration ${ }^{101}$. Interestingly, bulk chromatin, or 21 even a single lagging chromosome trapped in the cleavage furrow, can induce cytokinesis 22 failure and tetraploidization in human cells ${ }^{102,103}$. Cytokinesis defects have also been 23 reported in many different types of cancer cells (e.g. breast, colon and ovarian), as a result for 24 example of dysfunctions of various proteins that control the cytokinesis process (aurora A, 25 mitotic arrest-deficient 2 (MAD2) and large tumour suppressor 1 (LATS1)) ${ }^{21}$. 


\section{[H1] Polyploidy in the liver}

4 The liver is a vital organ with an extraordinary range of physiological functions, including the 5 metabolism of carbohydrates, lipids and amino acids, detoxification of xenobiotic compounds, synthesis of serum proteins, and bile production and secretion. These functions are performed primarily by hepatocytes, which account for $70 \%$ of the cells of the human liver ${ }^{104}$. Interestingly, the metabolic functions of hepatocytes are specific to their position

9 along the portocentral axis, making it possible to distinguish between 'periportal' hepatocytes 10 (which are involved in gluconeogenesis, ureagenesis, $\beta$-oxidation of fatty acids, amino acid breakdown and cholesterol biosynthesis) and 'pericentral' hepatocytes (which are involved in glycolysis, de novo lipogenesis and alcohol detoxification) ${ }^{105-109}$. Another source of hepatocyte heterogeneity is differences in ploidy. At birth, all hepatocytes are diploid, with a single nucleus containing two copies of each chromosome ${ }^{110,111}$. During postnatal growth, the liver parenchyma undergoes cellular changes characterized by gradual polyploidization, leading to the emergence of hepatocytes of several different ploidy classes (FIG. 3) ${ }^{110,111}$. Hepatocyte ploidy depends on the DNA content of each nucleus (nuclear ploidy: diploid, tetraploid, octoploid, and so on) and on the number of nuclei per hepatocyte (cellular ploidy: mononucleate, binucleate) ${ }^{110,111}$. For example, polyploid hepatocytes can be tetraploid (either binucleate with two diploid $(2 n)$ nuclei or mononucleate with a single tetraploid $(4 n)$ nucleus) or octoploid (either binucleate with two tetraploid (4n) nuclei or mononucleate with a single octoploid ( $8 n$ ) nucleus). The liver has long been known to display polyploidy, and various studies have determined the proportions of polyploid hepatocytes in the liver parenchyma of

24 different species ${ }^{112-116}$. For example, up to $90 \%$ of adult hepatocytes are polyploid in rodents $89,117-119$, versus approximately $30 \%$ in humans ${ }^{120-124}$. An increase in cell size is the most obvious and consistent consequence of an increase in ploidy. Several studies have shown that 27 a doubling of chromosome number approximately doubles the size of hepatocytes in humans 
1 and mice parenchyma ${ }^{125-128}$. There is no substantial difference in the volume of a given state

2 of ploidy, for example, between a tetraploid contingent of diploid binucleate cells $(2 \times 2 n)$ and

3 a tetraploid contingent of tetraploid mononucleate cells with a single tetraploid $(4 n)$ nucleus

4127,129 . Intriguingly, when the volume of the cell increases twofold during polyploidization,

5 the surface area of cellular structures increases only 1.4-fold (e.g. yeast and mammalian cells)

699 . In addition, as has already been demonstrated in Arabidopsis thaliana ${ }^{130}$, changes in the 7 proportions of cellular elements upon polyploidization, as chromosome movement, might 8 influence nuclear structure.

9

10

11

12

13

14

15

\section{[H1] Polyploidy in liver development}

Polyploidization during development has been analysed almost exclusively in rodent models.

All hepatocytes in the newborn rodent liver are diploid and have high rates of proliferation ${ }^{131}$. However, cell division gradually declines in rodents, such that, by six weeks of age, DNA synthesis is detected in only a few hepatocytes, at rates similar to those reported for normal adult liver ${ }^{89,132}$. Various in vivo studies have shown that polyploidization begins in the first few weeks after birth with the genesis of binucleate tetraploid hepatocytes $(2 \times 2 n)^{89,119,133-135}$. During this period, diploid hepatocytes $(2 n)$ follow either a normal cell cycle or an adapted cell cycle with karyokinesis but no cytokinesis (cytokinesis failure; FIG. 3). Proliferating binucleate tetraploid hepatocytes can then repeat DNA replication, leading either to cytokinesis, which generates two mononucleate tetraploid hepatocytes $(4 n)$, or to another failure of cytokinesis, which generates a binucleate octoploid hepatocyte $(2 \times 4 n)(\text { FIG. } 3)^{89}$. This situation results in the establishment of physiological polyploidy. Margall-Ducos et al. showed that the canonical cytokinesis programme is disrupted owing to the absence of actinomyosin ring formation ${ }^{134}$. Indeed, the organization of the actin cytoskeleton during the anaphase-telophase transition is impaired in these conditions. The microtubules fail to contact 
1 the cortex, and the molecular signals essential for cleavage plane specification (for example,

2 aurora B and polo-like kinase 1 (PLK1)) are therefore not correctly delivered. Consequently,

3 active RhoA, the principal orchestrator of cytokinesis, does not concentrate at the division

4 site, and the contractile ring is not formed ${ }^{134}$. During postnatal growth, cytokinesis failure

5 events occur around the time of weaning ${ }^{134}$ a period associated with profound changes in

6 feeding behaviour, hormone concentrations and metabolic pathways ${ }^{136}$. Indeed, insulin has

7 been identified as a key factor in the genesis of binucleate tetraploid hepatocytes ${ }^{137,138}$. In

8 rodent, impairment of insulin signalling greatly decreases the formation of binucleate

9 progenies, whereas repeated insulin injections promote the generation of polyploid liver cells.

10 The phosphoinositide 3-kinase (PI3K)-AKT pathway appears to be the principal downstream

11 pathway that mediates the cellular effects of insulin in these conditions. Various studies have

12 revealed that $\mathrm{PI} 3 \mathrm{~K}-\mathrm{AKT}$ regulates actin cytoskeleton polarization and reorganization during

13 processes such as cell migration and invasion ${ }^{139}$. The inhibition of AKT activity in dividing

14 hepatocytes induces the correct reorganization of actin during mitosis and the correct

15 localization of RhoA to the site of division, which is essential for successful cytokinesis ${ }^{137}$,

$16^{138}$. Other factors have also been shown to control binucleation during liver development. E2F

17 transcription factors, which are master regulators of cell cycle progression ${ }^{140}$, are also

18 essential. This family includes activators (E2F1, E2F2 and E2F3), repressors (E2F4, E2F5

19 and E2F6) and two atypical repressors (E2F7 and E2F8). In the mouse liver, E2F1-E2F6

20 levels are low throughout life, whereas E2F7 and E2F8 levels are high during the first seven

21 weeks after birth, a period that coincides with liver polyploidization ${ }^{119}$. E2f7 and E2f8-

22 knockout mouse livers were highly enriched with diploid hepatocytes $(60-70 \%$ of hepatocytes

23 in $\mathrm{KO}$ versus $3-4 \%$ in control livers $)^{65,119,141}$. A substantial portion of the deregulated

24 transcripts identified in E2f7 and E2f8-knockout mouse livers were involved in cytokinesis

25 (Ect2, Mklp1, Racgap1). Interestingly, during hepatocyte binucleation, Ect2, Mklp1 and 
1 Racgap are impaired in relaying information from the spindle to the cortex, thereby

2 preventing the formation of a functional contractile actomyosin ring and thus cytokinesis ${ }^{134}$.

3 E2F transcription factors have already been identified as key factors in the polyploidization

4 process in A. thaliana and D. melanogaster ${ }^{142-144}$. A similar phenomenon has been described 5 for trophoblast giant cells ${ }^{65}$ and endometrial stomal cells ${ }^{145}$ in mice. Interestingly, 6 microRNAs (miRNAs) are also drivers of physiological binucleation ${ }^{133}$. Mouse liver 7 miRNAs are expressed in an age-dependent manner, and a subset of these molecules, miR8122 in particular, is differentially expressed during postnatal development. Mir122 knockout 9 livers displayed $60 \%-70 \%$ reduction in the number of polyploid hepatocytes, this defect being 10 rescued by miR-122 overexpression ${ }^{133}$. During liver development, miR-122 directly 11 antagonizes pro-cytokinesis targets, thereby regulating hepatocyte binucleation ${ }^{133}$. Finally, 12 the silencing of cell cycle regulator genes, such as $C d k 1^{146}, \operatorname{Trp} 53^{147,148}, \operatorname{Cdkn} 1 a^{148,149}$, $13 \mathrm{cMyc}^{150}$, Ccne ${ }^{151}$, Birc5 (which encodes survivin) ${ }^{152}, \operatorname{Ssu} 72^{153}$, Stk3 (also known as Mst 1/2) $14^{154}, T g f b l^{155}$ and $R b 1^{148,156}$, has been shown to modify cellular or nuclear liver 15 polyploidization in various mouse models. However, the roles of these diverse genes in 16 controlling the cell cycle and division during liver development in relation to binucleation are unknown. Together, these studies highlight the essential contribution of cytokinesis failure to physiological liver polyploidization. Future studies should investigate how the combined activities of AKT, mTORC2, E2F7, E2F8 and miR-122 lead to inhibition of the cytokinesis machinery and the expansion of binucleate hepatocytes during liver development.

\section{[H1] Polyploidy during regeneration after tissues injuries}

24 The liver is continually exposed to tissue injuries and stresses throughout life. In the adult 25 human liver parenchyma, hepatocytes are slowly replaced, with each cell having a mean 26 lifespan of approximately $200-300$ days ${ }^{157}$, but they retain the ability to proliferate rapidly in 
1 response to aggression. The induction of liver regeneration by partial hepatectomy is a model

2 of compensatory hypertrophy, and several studies have revealed changes in ploidy profile

3 during this process. All hepatocytes enter the cell cycle after partial hepatectomy. By the end

4 of the regenerative process, cellular ploidy (binucleate polyploid hepatocytes) decreases, and

5 nuclear ploidy (mononucleate polyploid populations) increases (FIG. 4) ${ }^{158-162}$. Interestingly,

6 Wilkinson et al. demonstrated that the global ploidy spectrum remained unchanged after

7 complete liver regeneration in the partial hepatectomy mouse model ${ }^{141}$, which suggests that

8 the decrease in cellular ploidy is compensated for by the increase in nuclear ploidy. Miyaoka

9 et al. have used cell lineage approaches and a high-throughput imaging system to investigate

10 the mechanisms controlling polyploidization during mouse liver regeneration ${ }^{28}$. They found

11 that, although all hepatocytes entered into the cell cycle after partial hepatectomy, progressed

12 into $\mathrm{S}$ phase, only about half continue through mitosis suggesting that some hepatocytes

13 undergo endoreplication (amplification of nuclear ploidy). Importantly, hepatocytes that will

14 go into mitosis undergo cell division (genesis of mononucleate progenies). Thus, by contrast

15 to liver development, during liver regeneration, there is a shift from cellular polyploidy

16 (polyploid hepatocytes, predominantly binucleate) to nuclear polyploidy (polyploid

17 hepatocytes, predominantly mononucleate) (FIG. 4). Pathological conditions, such as hepatic

18 metabolic overload (of iron or copper) ${ }^{163-165}$, telomere attrition ${ }^{166}$ and chronic viral infection

19 (hepatitis $\mathrm{B}$ and $\mathrm{C}$ virus) $123,124,167$, also promote liver polyploidization, by as yet

20 uncharacterized mechanisms (FIG. 4). Polyploidy spectrum has been also characterized in

21 nonalcoholic fatty liver disease (NAFLD) (FIG. 4) ${ }^{31,168}$. In mice models of NAFLD, there

22 was an enrichment of highly polyploid mononucleate hepatocytes in the fatty liver

23 parenchyma. The same phenotype was observed in patients with nonalcoholic steatohepatitis.

24 The key question is, how are these cells generated? The cell cycles of normal hepatocytes

25 were compared with fatty hepatocytes in mouse primary cultures. Fatty hepatocytes 
1 progressed through G1 phase and entered S phase, but their progression through the S and G2

2 phases was delayed relative to control cells. This delay was associated with the activation of a

3 G2-M 'DNA damage signal' (ATR-p53-p21 pathway) that precludes the activation of the

4 mitotic kinase (CDK1-cyclin B) and leads to endoreplication ${ }^{31,168}$. This work led to the

5 identification of oxidative stress as a key driver of pathological polyploidization in the livers

6 of patients with NAFLD. These findings provided the first demonstration of the generation,

7 by endoreplication, of pathological polyploid hepatocytes in a damaged liver, through

8 activation of a DNA damage-signalling axis. It is tempting to speculate that endoreplication is

9 a favoured mode of liver tissue repair, but further studies are needed to determine how various

10 genotoxic stresses and cell cycle regulators act together to promote endoreplication, and also

11 the fate of the polyploid hepatocytes generated in such conditions. Emerging data from 12 multiple experimental models have suggested a conserved role for endoreplication as an 13 alternative means of proliferation in a context of genome instability ${ }^{57,67}$.

17 Is hepatocyte polyploidization merely a manifestation of the physiological growth or aggression of liver tissue, or do polyploid cells have functions that are physiologically relevant? The biological importance of liver polyploidization remains unclear, but various hypotheses have been proposed, some of which have already been partially validated.

\section{[H2] Polyploidization stage and terminal differentiation}

22 Pioneering studies in rodent and human have shown liver polyploidy to be related to terminal differentiation and senescence ${ }^{122,131,160,161,169-171}$. As a result, many studies have tried to

24 determine whether a polyploid hepatocyte can still divide in response to different proliferation 25 stimuli. Surprisingly, several groups argued that polyploid hepatocytes seem not to be engaged in senescence but by contrast maintained a high rate of proliferation. For instance, 
1 polyploid hepatocytes have been shown to divide as much as the diploid contingent after 2 partial hepatectomy ${ }^{28,172}$. The livers of $E 2 f 8^{-/-}$mice, which consist predominantly of diploid 3 hepatocytes, have a regenerative capacity similar to that of wild-type livers, which have a $4 \quad$ large proportion of polyploid cells ${ }^{119}$. Similarly, in mouse models of chronic liver injury 5 followed by competitive repopulation of hepatocytes, diploid and octoploid hepatocytes have 6 been shown to have equivalent repopulation potentials ${ }^{117,173}$. By contrast, other studies have 7 supported the idea that the polyploid state acts to suppress growth and restrict proliferation.

8 Ganem et al. reported that cytokinesis failure during postnatal murine liver development 9 triggers the Hippo-LATS2-p53 pathway, which restricts the growth of tetraploid hepatocytes $10{ }^{174}$. In addition, tetraploid murine hepatocyte growth has been shown to increase in a $\operatorname{Trp} 53^{-/-}$ 147, 174 . In 2018, Wilkinson et al. investigated whether diploid hepatocytes from wild-type 12 mouse livers had a proliferative advantage compared with polyploidy contingent ${ }^{141}$. They observed, both in vitro (primary mouse hepatocyte cultures) and in vivo (mouse liver regeneration assays), similar responses of diploid and polyploid hepatocytes to hepatic mitogens, indicating that proliferation kinetics are unrelated to differential responses to 16 growth stimuli. However, they also showed that diploid hepatocytes entered and completed the cell cycle more rapidly than their polyploid counterparts. Differences were also observed among polyploid hepatocytes, with tetraploid cells entering the cell cycle more rapidly than octoploids. The authors suggested that cell cycle regulation might subtly differ between diploid and polyploid contingents (for example, early replication licensing and mitosis 21 progression). The restrictive proliferation of the polyploid contingent might be related solely 22 to ageing. Diploid, tetraploid and octoploid hepatocytes from two-month-old mice have 23 identical capacities to repopulate the liver and do not express specific senescence markers, 24 such as p16, p21 and p53 ${ }^{175,176}$. However, as mice age, the expression of these senescence 25 markers becomes substantially stronger in octoploid than in diploid and tetraploid 
1 hepatocytes, and the repopulation potential of the octoploid contingent also decreases ${ }^{175,176}$.

2 Collectively, these data suggest that polyploid hepatocytes retain the ability to respond to

3 mitogen stimuli and to proliferate. However, during ageing, and possibly in some liver

4 diseases, polyploidy might induce senescence-type changes. Studies are therefore required to

5 improve our understanding of ploidy-specific proliferation in different liver injuries such as

6 chronic liver disease.

\section{$7 \quad$ [H2] Polyploidization as an enhancer of liver functions}

8 Polyploidy is a common strategy for organogenesis in various systems and organs including

9 the liver and, over the past 10 years, many studies in different experimental models have 10 highlighted specific functions for polyploid cells ${ }^{7,24,25,177}$. An innovative study by Rios and 11 co-workers revealed a role for specific mammalian polyploid cells ${ }^{178}$. They observed 12 binucleate polyploid cells in the lactating mammary glands of several different species (e.g. 13 humans, cows and mice) ${ }^{178}$. In mice, aurora kinase A (AURKA) and PLK1 control the 14 generation of these binucleate cells by cytokinesis failure at the time of the switch to lactation 15 in response to various signals, including prolactin and epidermal growth factor. The 16 binucleate alveolar cells were shown to be essential for lactation. Deletion of Aurka inhibited 17 the formation of binucleate cells and decreases milk synthesis ${ }^{178}$.

18 The liver participates in diverse functions, and it is tempting to speculate that physiologically 19 polyploid hepatocytes have their own features and roles. The gene expression profile in the 20 liver changes throughout the lobule (along the portocentral axis), resulting in a spatial 21 zonation of tasks ${ }^{105-109}$. Several groups have investigated whether polyploid hepatocytes 22 display a specific distribution within mouse hepatic lobules that might account for specific 23 functions, but the findings of these studies are conflicting. Some studies suggested that the 24 periportal region contains fewer polyploid hepatocytes than the perivenous region ${ }^{118,171}$, 25 whereas others suggested that the proportions of polyploid cells were similar in both regions 
119, 134. Two studies used tissue-imaging systems to reconstruct spatial zonation in the murine

2 liver and to quantify hepatocyte polyploidy ${ }^{129,179}$. They showed that the periportal and

3 perivenous regions were enriched in tetraploid hepatocytes but had a low density of octoploid

4 hepatocytes, whereas the mid-lobule region (equidistant between the periportal and

5 perivenous regions) displayed the opposite pattern: it was enriched in octoploid hepatocytes

6 but had a low density of tetraploid hepatocytes. They concluded that liver polyploidy

7 proceeds in spatial waves, advancing more rapidly in the mid-lobule region than in the

8 periportal and perivenous zones. One study investigated whether this zonation of polyploidy

9 was conserved in normal human hepatic lobules. Bou-Nader et al. observed that neither

10 mononucleate $(4 n$ or $\geq 8 n)$ nor binucleate $(2 \times 2 n$ or $\geq 2 \times 4 n)$ polyploid hepatocytes had a

11 specific zonal distribution ${ }^{120}$. Much less is known about metabolic zonation in the human

12 liver than in the mouse liver ${ }^{108}$. Single-cell in situ experiments will be required to analyse the

13 combined effects of metabolic zonation and polyploidy on hepatocyte fate.

14 A simple model used to explain the effects of polyploidy on cellular functions increases

15 transcription/traduction on a per cell basis (e.g. twice as many genes would produce twice as

16 many proteins) ${ }^{177,180,181}$. However, the situation is not quite that simple, as it has been

17 shown, in a number of species (e.g. plants, Drosophila, mammalian cells) that polyploidy

18 promotes nonuniform genome, transcriptome, epigenome and metabolome modifications ${ }^{177}$.

19 A few years ago, Lu et al. used a microarray approach to analyse the gene expression profile

20 of fluorescence-activated cell sorting (FACS)-isolated diploid, tetraploid and octoploid

21 hepatocytes from wild-type mice ${ }^{182}$. Surprisingly, only a few differences in gene expression

22 profile between the different populations were found. Kreutz and co-workers suggested that

23 gene expression profiles differ between diploid and mononucleate polyploid hepatocytes, but

24 not between diploid and binucleate polyploid hepatocytes ${ }^{183}$. This finding would suggest that

25 increases in nuclear ploidy in the liver parenchyma affect hepatocyte fate, a hypothesis that 
1 was tested in mice that lack the mitotic kinase CDK1. Following partial hepatectomy, Cdk1-

2 knockout livers regenerate fully, but hepatocytes endoreplicate, resulting in a large proportion

3 of mononucleate polyploid hepatocytes in the liver ${ }^{146}$. Using this model, Miettinen et al.

4 showed that higher ploidy levels were associated with lower levels of expression of

5 mitochondrial and de novo lipid biosynthesis genes and with higher levels of expression of

6 glycolysis genes ${ }^{181}$. The inhibition of mitochondrial functions and lipid synthesis increases

7 cell size in human epithelial cells, suggesting a possible causal link cellular metabolism and

8 polyploidy ${ }^{181}$. These results are consistent with the results of a comparative genome-scale

9 analysis of mouse and human liver tissues with different levels of polyploidy, in which highly

10 polyploid livers displayed anaerobic energy production, with ATP obtained from

11 carbohydrates rather than from fatty acids ${ }^{184,}{ }^{185}$. Collectively, these results suggest a

12 probable link between polyploidy and energy demands. Cells that display endoreplication or

13 cytokinesis failure can channel the energy that would have otherwise gone into cell division

14 (in particular, ATP and lipid membrane consumption) for other purposes ${ }^{99}$. Thus,

15 polyploidization might facilitate rapid adaptation to stresses and new-environmental cues.

16 Finally, Itzkovitz and collaborators demonstrated that liver polyploidy dampens the intrinsic

17 variability associated with transcriptional bursts ${ }^{186}$. In prokaryotes and eukaryotes, many

18 genes are transcribed in bursts, with stochastic production of mRNA from the transcription

19 sites, and with transcription switching between 'on' and 'off' states ${ }^{187}$. Using single molecule

20 fluorescence in situ hybridization (FISH), the authors demonstrated that gene expression in

21 the intact mouse liver consists of transcriptional bursts ${ }^{186}$. They observed that transcriptional

22 noise tends to be lower in tetraploid hepatocytes than in diploid hepatocytes. The authors

23 suggest that liver polyploidization might therefore counteract the noise increase caused by

24 genes becoming 'burstier' with age, leading to a more controlled gene expression. 


\section{1 [H2] Polyploidization creates genetic diversity}

2 Proliferating polyploid hepatocytes can increase their DNA content, but they can also reduce

3 it, through a process known as ploidy reversal ${ }^{117,121,188}$. Polyploid hepatocytes have larger

4 numbers of chromosomes than their diploid counterparts, but they also have supernumerary

5 centrosomes. For example, tetraploid hepatocytes (mononucleate or binucleate) have two

6 centrosomes in G1-S phases and four centrosomes in G2-M phases ${ }^{89}$. In most cases,

7 proliferating polyploid hepatocytes form a bipolar mitotic spindle through a specific

8 centrosome cluster (two centrosomes at each spindle pole), leading to the generation of

9 polyploid progenies (FIG. 4) ${ }^{89,117}$. Polyploid hepatocytes can also form multipolar mitotic

10 spindles and, in this case, cell division can lead to the generation of three or more daughter

11 cells with lower ploidy states than maternal cells (a phenomenon known as ploidy reversal)

12 (FIG. 4) ${ }^{117}$. The formation of a multipolar mitotic spindle is also linked to chromosome

13 segregation errors that arise from merotelic chromosome attachments (the attachment of one

14 kinetochore to both mitotic spindle poles). In this case, the division of polyploid hepatocytes

15 leads to random whole-chromosome gains and/or losses, resulting in aneuploid contingents

16117,121 . The connection between polyploidy, ploidy reversal and aneuploidy has been called

17 the 'ploidy conveyor' 110 . Interestingly, aneuploidy is observed in many different healthy

18 human tissues, including those of the skin, brain and placenta $117,121,188-191$. However, the

19 degree of aneuploidy in the healthy liver remains a matter of debate. Studies of proliferating

20 polyploid hepatocytes in primary mouse culture have revealed high rates of chromosome

21 segregation errors and aneuploidy (approximately 60\%) 117, 192. By contrast, single-cell

22 sequencing of hepatocytes in mouse and human liver tissues has revealed only a low level of

23 aneuploidy (about 5\%) ${ }^{188}$. An elegant study by Knouse and collaborators examine the

24 importance of the tissue environment for chromosome segregation fidelity in the polyploid

25 liver ${ }^{172}$. By comparing images of mitotic polyploid hepatocytes in mouse regenerating liver 
1 or in primary cultures, they demonstrated that tissue architecture is particularly important for

2 the correction of merotelic attachments and, therefore, for chromosome segregation fidelity in

3 the polyploid liver ${ }^{172}$. Recently, Marcus Grompe's group has developed a sophisticated

4 multicolor reporter allele system to genetically label and trace in situ mouse polyploid

5 hepatocytes in situ ${ }^{193}$. They observed that polyploid hepatocytes regenerate injured livers and

6 frequently reduce their ploidy. Ploidy-reduced progenies proliferate and keep the property to

7 increase their ploidy content. Importantly, they observed that during ploidy reduction

8 chromosome segregation is not random but faithful. All these data reinforce our view that the

9 ploidy conveyor model is intriguing, not least because it could generate genetic diversity in

10 the healthy liver. Duncan and co-workers have analysed the role of hepatic aneuploidy in

11 chronic liver injury, using $\mathrm{Fah}^{-/-}$mice, a well-known model of hereditary tyrosinaemia type 1

$12{ }^{194}$. In response to massive liver failure, hepatocytes with a partial loss of chromosome 16 13 expanded, repopulated the liver and restored liver function ${ }^{192}$. The partial loss of

14 chromosome 16, which carries the gene that encodes homogentisate 1, 2-dioxygenase, confers

15 resistance to the disease in this model of liver injury. Interestingly, a loss of polyploidy in the

16 liver (E2f7/E2f8 double knockout mouse model) resulted in decreased aneuploidy rates and 17 decreased the ability of the liver to adapt to tyrosinaemia-induced liver failure ${ }^{195}$. Further 18 studies are now required to determine whether ploidy reversal and specific aneuploid 19 hepatocytes can facilitate adaptation in chronic human liver diseases.

\section{0 [H2] Benefits of polyploidization for tissue repair}

21 Polyploidy can be benefic after acute organ failure in different organs, as shown in 22 Drosophila in particular. Losick and co-workers have shown that polyploidization contributes 23 to wound healing in adult D. melanogaster epithelia ${ }^{196}$. Indeed, the epithelial cells that 24 surround the injury site re-enter $\mathrm{S}$ phase, but, rather than dividing, these cells undergo 25 endocycling and become polyploid. In this case, epidermal repair is inhibited when 
1 polyploidy is blocked. In mammals, polyploidy can also be beneficial for tissue repair (e.g.

2 mouse, human) ${ }^{24,25,197}$. In an elegant study, Cao and co-workers devised genetic tools and

3 live imaging methods for visualizing cell-cycle dynamics during the regeneration of the

4 zebrafish epicardium ${ }^{26}$. Their results demonstrate that cells in the leading edge (called 'leader

5 cells') of the regenerating epicardium undergo endoreplication or cytokinesis failure and

6 generate polyploid cells. Farther from the lesion, 'follower cells' complete the cell cycle. An

7 increase in mechanical tension at the leading edge was found to be a key factor driving

8 polyploidy. Interestingly, most polyploid leader cells undergo apoptosis when regeneration is

9 complete. These results suggest that mechanical tension within a tissue can favour polyploidy

10 to support tissue regeneration ${ }^{26}$.

11 As the liver is the most robust regenerative organ in the human body and one of the best examples of polyploid tissues, it was tempting to speculate that the polyploid contingent has a major role in tissue repair. As described above, polyploid hepatocytes proliferate during mouse liver regeneration after partial hepatectomy. Surprisingly, these cells being as effective as diploid hepatocytes at restoring injured tissues ${ }^{28}$. This phenomenon has been analysed in 16 the liver of E2f7/E2f8 double knockout (LDKO) mice presenting reduced polyploidy. Resected polyploid livers (wild-type) support regeneration as effectively as resected predominantly diploid livers (LDKO) ${ }^{119,198}$. Furthermore, no difference in regeneration was observed when wild-type and LDKO livers were treated acutely with hepatotoxins, such as carbon tetrachloride or 3,5-diethoxycarnonyl-1,4-dihydrocollidine ${ }^{65,119}$. These results suggest that the restoration of liver size by polyploid cell growth during liver regeneration after partial hepatectomy or after acute injury is not beneficial. Future studies will determine whether polyploidization is beneficial for tissue repair following chronic liver injury. 
1 Polyploid cells have been observed in multiple cancer types such as pancreatic ${ }^{199}$, cervical ${ }^{53}$,

2 lung ${ }^{200}$, colon ${ }^{201}$ and prostate ${ }^{202}$. Genomic studies have revealed that approximately $30 \%$ of

3 solid tumours have polyploid or near-polyploid karyotypes ${ }^{36,203}$. Clinically, polyploidy has

4 been associated with aggressive, difficult-to-treat different solid tumours (e.g. colorectal and

5 pancreatic cancers) ${ }^{80,203,204}$. Numerous studies have observed that polyploid cells are present

6 from the transition from premalignant to malignant disease suggesting that a genome-

7 multiplying event can be a driver of tumorigenesis ${ }^{53,205,206}$. It is therefore not surprising that

8 mechanisms have evolved to limit the proliferation of these cells. It is well illustrated that the

9 p53 pathway limits the proliferation of polyploid cells to protect genomic integrity $30,207,208$.

10 Polyploid cancer cells (e.g. colorectal, fibrosarcoma) can be also recognized by the immune 11 system in such a way that their growth is suppressed or delayed 74,209 . It has been 12 demonstrated that $\mathrm{CD} 4^{+}$and $\mathrm{CD}^{+} \mathrm{T}^{\mathrm{T}}$ lymphocytes, the interferon system ${ }^{210}$ and NK cells ${ }^{211}$ 13 are involved in the control of polyploid cells. One of the mechanism through which polyploid 14 cells can be recognized by the immune system implies an endoplasmic reticulum (ER) stress 15 response resulting in the exposure of the 'eat-me' signal calreticulin (CALR) at the cell 16 surface ${ }^{210,212}$. Senovilla and collaborators also demonstrated that polyploid mouse colon 17 cancer readily proliferated in immunodeficient mice and conserved their increased DNA 18 content ${ }^{210}$. Interestingly, the same cells injected into immunocompetent mice generated 19 tumours only after a delay, and such tumours exhibited reduced DNA content, endoplasmic 20 reticulum stress and calreticulin exposure ${ }^{210}$.

21 The accepted model is that tetraploidy drives carcinogenesis by acting as a stochastic 22 generator of genomic instability. As described earlier, polyploid cells have supernumerary 23 centrosomes, and this feature is linked to high rates of chromosome missegregation in mitosis $24207,213,214$. Also, one study neatly showed in colorectal cancer cell lines that tetraploidy 25 induces replication stress through a massive deregulation of genes that are involved in the 
1 correct functioning of the DNA replication machinery ${ }^{215}$. Replication stress has emerged as a

2 source of $\mathrm{CIN}^{216}$. Several laboratories working on different experimental models have shown

3 that polyploidy is a gateway state that participate to tumorigenesis ${ }^{34,70,204,217}$. The pioneering

4 experiments were performed by David Pellman and colleagues who showed that TP53 ${ }^{-/-}$

5 tetraploid mammary epithelial cells gave rise to malignant tumours in nude mice, whereas

6 TP53 $^{-/}$diploid mammary epithelial cells did not ${ }^{34,217}$. This finding led to the suggestion that

7 tetraploidy causes tumorigenesis. The temporal relationship between TP53 mutations and

8 polyploidy has been analysed in tumours from prospectively characterized patients with

9 advanced cancers (e.g. colorectal, pancreatic, lung, uterine) ${ }^{203}$. As expected, polyploidy was

10 statistically associated with TP53-mutated tumours. Interestingly, telomerase reverse

11 transcriptase (TERT) promoter mutations are associated with an E2F-mediated G1 arrest

12 defect, but not with polyploidization ${ }^{203}$. Overall, these data suggest that polyploidization is a

13 key step in tumour formation and progression.

14 The link between polyploidy and the origin of cancer has primarily been demonstrated in 15 tissues that are physiologically diploid. The role of polyploidy during liver tumorigenesis is 16 quite puzzling, owing to the physiologically polyploid state of normal adult liver. Whether 17 polyploidy is a risk factor for or protective against malignant transformation was until 18 recently unknown. The most widely-accepted hypothesis is that liver polyploidy acts as a 19 gatekeeper of tumorigenesis ${ }^{218}$. In theory, physiological polyploidization could protect cells 20 against genotoxic damage by increasing gene copy numbers per cell, which could at least 21 buffer gene-inactivating mutations ${ }^{22,177}$. We can easily imagine that in a diploid cell the loss 22 of heterozygosity of a tumour suppressor gene could have dramatic consequences, such as 23 uncontrolled proliferation. Acquiring multiple sets of chromosomes could provide backup 24 tumour suppressor gene copies. In a series of elegant experiments, Zhang et al. demonstrated 25 that polyploid mouse livers were protected from tumour-suppressor loss of heterozygosity ${ }^{198}$. 
1 To modify the liver ploidy spectrum, two genes were silenced: Anln, an actin-binding protein

2 required for cytokinesis, and E2f8, which is required for liver polyploidization. Ploidy was

3 substantially increased after Anln knockdown (livers with the majority of hepatocytes are

4 polyploid) and decreased after E2f8 knockdown (livers with the majority of hepatocytes are

5 diploid). The authors observed that higher levels of polyploidy reduced tumour incidence in

6 diverse mouse liver cancer models, suggesting that polyploidy helps to prevent tumour

7 development. Notably, in this study, ploidy content has been modified in non-tumoural liver

8 tissue with wild-type $\operatorname{Tr} 553$. As demonstrated in others contexts, it is likely that polyploidy in

9 the absence of $\operatorname{Trp} 53$ could be a driver of liver tumorigenesis. The presence of polyploid cells

10 within liver tumours could also act as a driver of tumour progression. In 2019, an in situ

11 imaging approach was developed to determine whether cellular and nuclear ploidy content is

12 altered during human liver tumorigenesis ${ }^{120}$. The ploidy spectra of surgically resected tissues

13 from patients with hepatocellular carcinoma (HCC) as well as from healthy controls were

14 determined. These data show that the binucleate polyploid fraction, or cellular ploidy, is

15 drastically reduced during human liver tumorigenesis $(\approx 15 \%$ in normal tissue versus $5 \%$ in

16 tumoral tissue). Conversely, the mononucleate polyploid fraction, or nuclear ploidy, is

17 amplified in HCCs $(\approx 12 \%$ in normal tissue versus $33 \%$ in tumoral tissue). Next, Bou-Nader et

18 al. compared nuclear ploidy spectra on the basis of histological and molecular features of

19 HCC tumours. Nuclear ploidy was sufficient to distinguish between premalignant and

20 malignant liver parenchyma. Mononucleate polyploid hepatocytes were enriched in HCCs

21 that harboured a low grade of differentiation, a high proliferation rate and a poor prognosis.

22 Furthermore, it was observed that TP53 mutations account for a higher percentage of

23 mononucleate polyploid hepatocytes than both TERT promoter-mutated and CTNNB1-

24 mutated HCCs. 
1 Taken together, these results indicate that polyploid hepatocytes act as a kind of 'Jekyll and

2 Hyde' during liver tumorigenesis (FIG. 5). A polyploid state could provide protection from

3 tumorigenesis by providing extra copies of tumour suppressor genes and by restricting

4 hepatocyte proliferation in pre-malignant tissue with wild-type TP53. Conversely, 5 amplification of mononucleate polyploid hepatocytes within HCCs is associated with TP53 6 mutations, a high proliferative rate and a poor prognosis.

7

[H1] Conclusions

9

Polyploidization is a fascinating mechanism that is essential for tissue homeostasis but also has a role in tumorigenesis. Studies have revealed that the liver is one of the few mammalian organs to display changes in ploidy in various circumstances. In physiological conditions, progressive polyploidization occurs during liver development and in ageing, indicating terminal differentiation. We have learned much about the generation of polyploid hepatocytes through cytokinesis failure, but we still have much to learn about the function of these cells in liver homeostasis.

17 There are still important questions that need to be addressed. Is there a difference in cellular states between diploid and polyploid hepatocytes (that is, in epigenetics, transcriptomes, proteomes, and so on)? Is there a limit to hepatocyte ploidy? How do polyploid hepatocytes cope with genome copy number variation and centrosome amplification? And finally, what are the molecular cues of the ploidy conveyor, and does liver aneuploidy affect human health? The liver parenchyma displays changes in ploidy following tissue injury or stress, particularly in the context of NAFLD. Future studies should aim to understand whether pathological 24 polyploidization takes place in other pathologies, and to determine how these polyploid 25 contingents behave in damaged livers. Do they have specific lobule zonation? Do they influence disease progression? It will be particularly interesting to determine whether 
1 hepatocyte polyploidization affects the process of liver infection (for example, of HCV and

$2 \mathrm{HBV}$ ). Interestingly, Plasmodium parasites have been shown to preferentially infect and 3 develop in polyploid hepatocytes ${ }^{219}$.

4 Finally, a major challenge in this field will be to determine whether polyploid HCCs derive 5 from polyploid or diploid hepatocytes. If they are derived from polyploid hepatocytes, then 6 the use of polyploidization to protect the liver would be dangerous. Conversely, if polyploid 7 tumours arise from diploid hepatocytes, then the polyploid state might provide protection in 8 pathological settings by providing extra copies of tumour suppressor genes and restricting 9 hepatocyte proliferation. 


\section{References}

1. O'Brien, S.J., Menninger, J.C. \& Nash, W.G. in Acta Physiol (Oxf) (2006).

2. Otto, S.P. The evolutionary consequences of polyploidy. Cell 131, 452-62 (2007).

3. Weaver, B.A. \& Cleveland, D.W. Does aneuploidy cause cancer? Curr Opin Cell Biol 18, 658-67 (2006).

4. Gallagher, J.P., Grover, C.E., Hu, G. \& Wendel, J.F. Insights into the Ecology and Evolution of Polyploid Plants through Network Analysis. Mol Ecol 25, 2644-60 (2016).

5. Dehal, P. \& Boore, J.L. Two rounds of whole genome duplication in the ancestral vertebrate. PLoS Biol 3, e314 (2005).

6. Soltis, D.E., Visger, C.J. \& Soltis, P.S. The polyploidy revolution then....and now: Stebbins revisited. Am J Bot 101, 1057-1078 (2014).

7. Van de Peer, Y., Mizrachi, E. \& Marchal, K. The evolutionary significance of polyploidy. Nat Rev Genet 18, 411-424 (2017).

8. Leitch, A.R. \& Leitch, I.J. Genomic plasticity and the diversity of polyploid plants. Science 320, 481-3 (2008).

9. Otto, S.P. \& Whitton, J. Polyploid incidence and evolution. Annu Rev Genet 34, 401-437 (2000).

10. Ramsey, J. \& Schemske, D.W. Pathways, mechanisms and rates of polyploid formation in flowering plants. Annu. Rev. Ecol. Syst. 29, 467-501 (1998).

11. Jiao, Y. et al. Ancestral polyploidy in seed plants and angiosperms. Nature 473, 97-100 (2011).

12. Soltis, D.E., Bell, C.D., Kim, S. \& Soltis, P.S. Origin and early evolution of angiosperms. Ann N Y Acad Sci 1133, 3-25 (2008).

13. Soltis, P.S. \& Soltis, D.E. Ancient WGD events as drivers of key innovations in angiosperms. Curr Opin Plant Biol 30, 159-65 (2016).

14. Mable, B., Alexandrou, M.A. \& Taylor, M.I. Genome duplication in amphibians and fish: an extended synthesis. Journal of Zoology 284, 151-182 (2011).

15. Guc-Scekic, M., Milasin, J., Stevanovic, M., Stojanov, L.J. \& Djordjevic, M. Tetraploidy in a 26-month-old girl (cytogenetic and molecular studies). Clin Genet 61, 62-5 (2002).

16. Jacobs, P.A., Szulman, A.E., Funkhouser, J., Matsuura, J.S. \& Wilson, C.C. Human triploidy: relationship between parental origin of the additional haploid complement and development of partial hydatidiform mole. Ann Hum Genet 46, 223-31 (1982).

17. Wertheim, B., Beukeboom, L.W. \& van de Zande, L. Polyploidy in animals: effects of gene expression on sex determination, evolution and ecology. Cytogenet Genome Res 140, 256-69 (2013).

18. Gallardo, M.H., Bickham, J.W., Honeycutt, R.L., Ojeda, R.A. \& Kohler, N. Discovery of tetraploidy in a mammal. Nature 401, 341 (1999).

19. Anzi, S. et al. Postnatal Exocrine Pancreas Growth by Cellular Hypertrophy Correlates with a Shorter Lifespan in Mammals. Dev Cell 45, 726-737 e3 (2018).

20. Oates, P.S. \& Morgan, R.G. Changes in pancreatic acinar cell nuclear number and DNA content during aging in the rat. Am J Anat 177, 547-54 (1986).

21. Davoli, T. \& de Lange, T. The causes and consequences of polyploidy in normal development and cancer. Annu Rev Cell Dev Biol 27, 585-610 (2011). 
22. Pandit, S.K., Westendorp, B. \& de Bruin, A. Physiological significance of polyploidization in mammalian cells. Trends Cell Biol 23, 556-66 (2013).

23. Ravid, K., Lu, J., Zimmet, J.M. \& Jones, M.R. Roads to polyploidy: the megakaryocyte example. J Cell Physiol 190, 7-20. (2002).

24. Gjelsvik, K.J., Besen-McNally, R. \& Losick, V.P. Solving the Polyploid Mystery in Health and Disease. Trends Genet 35, 6-14 (2019).

25. Ovrebo, J.I. \& Edgar, B.A. Polyploidy in tissue homeostasis and regeneration. Development 145 (2018).

26. Cao, J. et al. Tension Creates an Endoreplication Wavefront that Leads Regeneration of Epicardial Tissue. Dev Cell 42, 600-615 e4 (2017).

27. Lazzeri, E. et al. Endocycle-related tubular cell hypertrophy and progenitor proliferation recover renal function after acute kidney injury. Nat Commun 9, 1344 (2018).

28. Miyaoka, Y. et al. Hypertrophy and unconventional cell division of hepatocytes underlie liver regeneration. Curr Biol 22, 1166-75 (2012).

29. Auer, G.U., Backdahl, M., Forsslund, G.M. \& Askensten, U.G. Ploidy levels in nonneoplastic and neoplastic thyroid cells. Anal Quant Cytol Histol 7, 97-106 (1985).

30. Ganem, N.J., Storchova, Z. \& Pellman, D. Tetraploidy, aneuploidy and cancer. Curr Opin Genet Dev 17, 157-62 (2007).

31. Gentric, G. et al. Oxidative stress promotes pathologic polyploidization in nonalcoholic fatty liver disease. J Clin Invest 125, 981-92 (2015).

32. Hixon, M.L. \& Gualberto, A. Vascular smooth muscle polyploidization--from mitotic checkpoints to hypertension. Cell Cycle 2, 105-10 (2003).

33. Vliegen, H.W., Eulderink, F., Bruschke, A.V., van der Laarse, A. \& Cornelisse, C.J. Polyploidy of myocyte nuclei in pressure overloaded human hearts: a flow cytometric study in left and right ventricular myocardium. Am J Cardiovasc Pathol 5, 27-31 (1995).

34. Fujiwara, T. et al. Cytokinesis failure generating tetraploids promotes tumorigenesis in p53-null cells. Nature 437, 1043-7 (2005).

35. Jamal-Hanjani, M. et al. Tracking the Evolution of Non-Small-Cell Lung Cancer. $N$ Engl J Med 376, 2109-2121 (2017).

36. Zack, T.I. et al. Pan-cancer patterns of somatic copy number alteration. Nat Genet 45, 1134-1140 (2013).

37. Yanagimachi, R. \& Noda, Y.D. Physiological changes in the postnuclear cap region of mammalian spermatozoa: a necessary preliminary to the membrane fusion between sperm and egg cells. J Ultrastruct Res 31, 486-93 (1970).

38. Huppertz, B., Frank, H.G., Kingdom, J.C., Reister, F. \& Kaufmann, P. Villous cytotrophoblast regulation of the syncytial apoptotic cascade in the human placenta. Histochem Cell Biol 110, 495-508 (1998).

39. Loutit, J.F. \& Nisbet, N.W. The origin of osteoclasts. Immunobiology 161, 193-203 (1982).

40. Taylor, M.V. Muscle differentiation: how two cells become one. Curr Biol 12, R224-8 (2002).

41. Abmayr, S.M. \& Pavlath, G.K. Myoblast fusion: lessons from flies and mice. Development 139, 641-56 (2012).

42. Kim, J.H., Jin, P., Duan, R. \& Chen, E.H. Mechanisms of myoblast fusion during muscle development. Curr Opin Genet Dev 32, 162-70 (2015). 
43. Lluis, F. \& Cosma, M.P. Cell-fusion-mediated somatic-cell reprogramming: a mechanism for tissue regeneration. J Cell Physiol 223, 6-13 (2010).

44. Alvarez-Dolado, M. et al. Fusion of bone-marrow-derived cells with Purkinje neurons, cardiomyocytes and hepatocytes. Nature 425, 968-73 (2003).

45. Rizvi, A.Z. et al. Bone marrow-derived cells fuse with normal and transformed intestinal stem cells. Proc Natl Acad Sci U S A 103, 6321-5 (2006).

46. Sanges, D. et al. Wnt/beta-catenin signaling triggers neuron reprogramming and regeneration in the mouse retina. Cell Rep 4, 271-86 (2013).

47. Wang, X. et al. Cell fusion is the principal source of bone-marrow-derived hepatocytes. Nature 422, 897-901 (2003).

48. Weimann, J.M., Johansson, C.B., Trejo, A. \& Blau, H.M. Stable reprogrammed heterokaryons form spontaneously in Purkinje neurons after bone marrow transplant. Nat Cell Biol 5, 959-66 (2003).

49. Duelli, D. \& Lazebnik, Y. Cell-to-cell fusion as a link between viruses and cancer. Nat Rev Cancer 7, 968-76 (2007).

50. Moody, C.A. \& Laimins, L.A. Human papillomavirus oncoproteins: pathways to transformation. Nat Rev Cancer 10, 550-60 (2010).

51. Gao, P. \& Zheng, J. High-risk HPV E5-induced cell fusion: a critical initiating event in the early stage of HPV-associated cervical cancer. Virol J 7, 238 (2010).

52. $\mathrm{Hu}, \mathrm{L}$. et al. Human papillomavirus $16 \mathrm{E} 5$ induces bi-nucleated cell formation by cell-cell fusion. Virology 384, 125-34 (2009).

53. Olaharski, A.J. et al. Tetraploidy and chromosomal instability are early events during cervical carcinogenesis. Carcinogenesis 27, 337-43 (2006).

54. Olaharski, A.J. \& Eastmond, D.A. Elevated levels of tetraploid cervical cells in human papillomavirus-positive Papanicolaou smears diagnosed as atypical squamous cells of undetermined significance. Cancer 102, 192-9 (2004).

55. Hartwell, L.H. \& Weinert, T.A. Checkpoints: controls that ensure the order of cell cycle events. Science 246, 629-34 (1989).

56. Edgar, B.A. \& Orr-Weaver, T.L. Endoreplication cell cycles: more for less. Cell 105, 297-306 (2001).

57. Fox, D.T. \& Duronio, R.J. Endoreplication and polyploidy: insights into development and disease. Development 140, 3-12 (2013).

58. Lee, H.O., Davidson, J.M. \& Duronio, R.J. Endoreplication: polyploidy with purpose. Genes Dev 23, 2461-77 (2009).

59. Orr-Weaver, T.L. When bigger is better: the role of polyploidy in organogenesis. Trends Genet 31, 307-15 (2015).

60. Edgar, B.A. \& Orr-Weaver, T.L. Endoreplication cell cycles: more for less. Cell 105, 297-306. (2001).

61. Edgar, B.A., Zielke, N. \& Gutierrez, C. Endocycles: a recurrent evolutionary innovation for post-mitotic cell growth. Nat Rev Mol Cell Biol 15, 197-210 (2014).

62. Hu, D. \& Cross, J.C. Development and function of trophoblast giant cells in the rodent placenta. Int J Dev Biol 54, 341-54 (2010).

63. Ogawa, H. et al. Cell proliferation potency is independent of FGF4 signaling in trophoblast stem cells derived from androgenetic embryos. J Reprod Dev 62, 51-8 (2016).

64. Ullah, Z., Kohn, M.J., Yagi, R., Vassilev, L.T. \& DePamphilis, M.L. Differentiation of trophoblast stem cells into giant cells is triggered by p57/Kip2 inhibition of CDK1 activity. Genes Dev 22, 3024-36 (2008). 
65. Chen, H.Z. et al. Canonical and atypical E2Fs regulate the mammalian endocycle. Nat Cell Biol 14, 1192-202 (2012).

66. Ouseph, M.M. et al. Atypical E2F repressors and activators coordinate placental development. Dev Cell 22, 849-62 (2012).

67. Shu, Z., Row, S. \& Deng, W.M. Endoreplication: The Good, the Bad, and the Ugly. Trends Cell Biol 28, 465-474 (2018).

68. Adachi, M. \& Brenner, D.A. High molecular weight adiponectin inhibits proliferation of hepatic stellate cells via activation of adenosine monophosphateactivated protein kinase. Hepatology 47, 677-85 (2008).

69. Radziejwoski, A. et al. Atypical E2F activity coordinates PHR1 photolyase gene transcription with endoreduplication onset. EMBO J 30, 355-63 (2011).

70. Davoli, T. \& de Lange, T. Telomere-driven tetraploidization occurs in human cells undergoing crisis and promotes transformation of mouse cells. Cancer Cell 21, 765-76 (2012).

71. Davoli, T., Denchi, E.L. \& de Lange, T. Persistent telomere damage induces bypass of mitosis and tetraploidy. Cell 141, 81-93 (2010).

72. Balachandran, R.S. \& Kipreos, E.T. Addressing a weakness of anticancer therapy with mitosis inhibitors: Mitotic slippage. Mol Cell Oncol 4, e1277293 (2017).

73. Mishima, M., Pavicic, V., Gruneberg, U., Nigg, E.A. \& Glotzer, M. Cell cycle regulation of central spindle assembly. Nature 430, 908-13 (2004).

74. Vitale, I., Galluzzi, L., Castedo, M. \& Kroemer, G. Mitotic catastrophe: a mechanism for avoiding genomic instability. Nat Rev Mol Cell Biol 12, 385-92 (2011).

75. Rieder, C.L. \& Maiato, H. Stuck in division or passing through: what happens when cells cannot satisfy the spindle assembly checkpoint. Dev Cell 7, 637-51 (2004).

76. Sinha, D., Duijf, P.H.G. \& Khanna, K.K. Mitotic slippage: an old tale with a new twist. Cell Cycle 18, 7-15 (2019).

77. Brito, D.A. \& Rieder, C.L. Mitotic checkpoint slippage in humans occurs via cyclin B destruction in the presence of an active checkpoint. Curr Biol 16, 1194-200 (2006).

78. Lee, J., Kim, J.A., Margolis, R.L. \& Fotedar, R. Substrate degradation by the anaphase promoting complex occurs during mitotic slippage. Cell Cycle 9, 1792801 (2010).

79. Balachandran, R.S. et al. The ubiquitin ligase CRL2ZYG11 targets cyclin B1 for degradation in a conserved pathway that facilitates mitotic slippage. J Cell Biol 215, 151-166 (2016).

80. Coward, J. \& Harding, A. Size Does Matter: Why Polyploid Tumor Cells are Critical Drug Targets in the War on Cancer. Front Oncol 4, 123 (2014).

81. Dikovskaya, D. et al. Loss of APC induces polyploidy as a result of a combination of defects in mitosis and apoptosis. J Cell Biol 176, 183-95 (2007).

82. Kinzler, K.W. \& Vogelstein, B. Lessons from hereditary colorectal cancer. Cell 87, 159-70 (1996).

83. Caldwell, C.M. \& Kaplan, K.B. The role of APC in mitosis and in chromosome instability. Adv Exp Med Biol 656, 51-64 (2009).

84. D'Avino, P.P., Giansanti, M.G. \& Petronczki, M. Cytokinesis in animal cells. Cold Spring Harb Perspect Biol 7, a015834 (2015).

85. Celton-Morizur, S. \& Desdouets, C. Polyploidization of liver cells. Adv Exp Med Biol 676, 123-135 (2010). 
86. Fortier, M., Celton-Morizur, S. \& Desdouets, C. Incomplete cytokinesis/binucleation in mammals: The powerful system of hepatocytes. Methods Cell Biol 137, 119-142 (2017).

87. Li, F., Wang, X., Bunger, P.C. \& Gerdes, A.M. Formation of binucleated cardiac myocytes in rat heart: I. Role of actin-myosin contractile ring. J Mol Cell Cardiol 29, 1541-51 (1997).

88. Zimmet, J. \& Ravid, K. Polyploidy: occurrence in nature, mechanisms, and significance for the megakaryocyte-platelet system. Exp Hematol 28, 3-16 (2000).

89. Guidotti, J.E. et al. Liver cell polyploidization: a pivotal role for binuclear hepatocytes. J Biol Chem 278, 19095-101 (2003).

90. Li, F., Wang, X., Capasso, J.M. \& Gerdes, A.M. Rapid transition of cardiac myocytes from hyperplasia to hypertrophy during postnatal development. J Mol Cell Cardiol 28, 1737-46 (1996).

91. Soonpaa, M.H., Kim, K.K., Pajak, L., Franklin, M. \& Field, L.J. Cardiomyocyte DNA synthesis and binucleation during murine development. Am J Physiol 271, H2183-9 (1996).

92. Bergmann, 0. et al. Dynamics of Cell Generation and Turnover in the Human Heart. Cell 161, 1566-75 (2015).

93. Yuan, X. \& Braun, T. Multimodal Regulation of Cardiac Myocyte Proliferation. Circ Res 121, 293-309 (2017).

94. Mollova, M. et al. Cardiomyocyte proliferation contributes to heart growth in young humans. Proc Natl Acad Sci U S A 110, 1446-51 (2013).

95. Liu, Z., Yue, S., Chen, X., Kubin, T. \& Braun, T. Regulation of cardiomyocyte polyploidy and multinucleation by CyclinG1. Circ Res 106, 1498-506 (2010).

96. Engel, F.B., Schebesta, M. \& Keating, M.T. Anillin localization defect in cardiomyocyte binucleation. J Mol Cell Cardiol 41, 601-12 (2006).

97. Ahuja, P., Sdek, P. \& MacLellan, W.R. Cardiac myocyte cell cycle control in development, disease, and regeneration. Physiol Rev 87, 521-44 (2007).

98. Storchova, Z. \& Kuffer, C. The consequences of tetraploidy and aneuploidy. J Cell Sci 121, 3859-66 (2008).

99. Storchova, Z. \& Pellman, D. From polyploidy to aneuploidy, genome instability and cancer. Nat Rev Mol Cell Biol 5, 45-54 (2004).

100. Tormos, A.M., Talens-Visconti, R. \& Sastre, J. Regulation of cytokinesis and its clinical significance. Crit Rev Clin Lab Sci 52, 159-67 (2015).

101. Lacroix, B. \& Maddox, A.S. Cytokinesis, ploidy and aneuploidy. J Pathol 226, 33851 (2012).

102. Mullins, J.M. \& Biesele, J.J. Terminal phase of cytokinesis in D-98s cells. J Cell Biol 73, 672-84 (1977).

103. Shi, Q. \& King, R.W. Chromosome nondisjunction yields tetraploid rather than aneuploid cells in human cell lines. Nature 437, 1038-42 (2005).

104. Si-Tayeb, K., Lemaigre, F.P. \& Duncan, S.A. Organogenesis and development of the liver. Dev Cell 18, 175-89 (2010).

105. Benhamouche, S. et al. Apc tumor suppressor gene is the "zonation-keeper" of mouse liver. Dev Cell 10, 759-70 (2006).

106. Gebhardt, R. Metabolic zonation of the liver: regulation and implications for liver function. Pharmacol Ther 53, 275-354 (1992).

107. Jungermann, K. \& Kietzmann, T. Zonation of parenchymal and nonparenchymal metabolism in liver. Annu Rev Nutr 16, 179-203 (1996). 
108. Ben-Moshe, S. \& Itzkovitz, S. Spatial heterogeneity in the mammalian liver. Nat Rev Gastroenterol Hepatol (2019).

109. Halpern, K.B. et al. Single-cell spatial reconstruction reveals global division of labour in the mammalian liver. Nature 542, 352-356 (2017).

110. Duncan, A.W. Aneuploidy, polyploidy and ploidy reversal in the liver. Semin Cell Dev Biol (2013).

111. Gentric, G. \& Desdouets, C. Polyploidization in liver tissue. Am J Pathol 184, 32231 (2014).

112. Brodsky, W.Y. \& Uryvaeva, I.V. Cell polyploidy: its relation to tissue growth and function. Int Rev Cytol 50, 275-332 (1977).

113. Carriere, R. Polyploid cell reproduction in normal adult rat liver. Exp. Cell. Res. 46, 533-540 (1967).

114. Nadal, C. \& Zajdela, F. [Polyploidy in the rat liver. II. The role of the hypophysis and protein deficiency]. Exp Cell Res 42, 117-29 (1966).

115. Nadal, C. \& Zajdela, F. [Hepatic polyploidy in the rat. IV. Experimental changes in the nucleolar volume of liver cells and their mechanisms of regulation]. Exp Cell Res 48, 518-28. (1967).

116. Anatskaya, O.V., Vinogradov, A.E. \& Kudryavtsev, B.N. Hepatocyte polyploidy and metabolism/life-history traits: hypotheses testing. J Theor Biol 168, 191-9 (1994).

117. Duncan, A.W. et al. The ploidy conveyor of mature hepatocytes as a source of genetic variation. Nature 467, 707-10 (2010).

118. Gandillet, A. et al. Hepatocyte ploidy in normal young rat. Comp Biochem Physiol A Mol Integr Physiol 134, 665-73 (2003).

119. Pandit, S.K. et al. E2F8 is essential for polyploidization in mammalian cells. Nat Cell Biol 14, 1181-91 (2012).

120. Bou-Nader, M. et al. Polyploidy spectrum: a new marker in HCC classification. Gut (2019).

121. Duncan, A.W. et al. Frequent aneuploidy among normal human hepatocytes. Gastroenterology 142, 25-8 (2012).

122. Kudryavtsev, B.N., Kudryavtseva, M.V., Sakuta, G.A. \& Stein, G.I. Human hepatocyte polyploidization kinetics in the course of life cycle. Virchows Arch $B$ Cell Pathol Incl Mol Pathol 64, 387-93 (1993).

123. Toyoda, H. et al. Changes to hepatocyte ploidy and binuclearity profiles during human chronic viral hepatitis. Gut 54, 297-302 (2005).

124. Toyoda, H., Kumada, T., Bregerie, O., Brechot, C. \& Desdouets, C. Conserved balance of hepatocyte nuclear DNA content in mononuclear and binuclear hepatocyte populations during the course of chronic viral hepatitis. World $J$ Gastroenterol 12, 4546-8 (2006).

125. Deschenes, J., Valet, J.P. \& Marceau, N. The relationship between cell volume, ploidy. and functional activity in differentiating hepatocytes. Cell Biophys 3, 32134 (1981).

126. Epstein, C.J. Cell size, nuclear content and the development of polyploidy in the mammalian liver. Proc Natl Acad Sci U S A 57, 327-334 (1967).

127. Martin, N.C. et al. Functional analysis of mouse hepatocytes differing in DNA content: volume, receptor expression, and effect of IFNgamma. J Cell Physiol 191, 138-44 (2002). 
128. Watanabe, T. \& Tanaka, Y. Age-related alterations in the size of human hepatocytes. A study of mononuclear and binucleate cells. Virchows Arch B Cell Pathol Incl Mol Pathol 39, 9-20 (1982).

129. Morales-Navarrete, $H$. et al. A versatile pipeline for the multi-scale digital reconstruction and quantitative analysis of 3D tissue architecture. Elife 4 (2015).

130. Kato, N. \& Lam, E. Chromatin of endoreduplicated pavement cells has greater range of movement than that of diploid guard cells in Arabidopsis thaliana. J Cell Sci 116, 2195-201 (2003).

131. Gupta, S. Hepatic polyploidy and liver growth control. Semin Cancer Biol 10, 16171 (2000).

132. Viola-Magni, M.P. Synthesis and turnover of DNA in hepatocytes of neonatal rats. $J$ Microsc 96, 191-203 (1972).

133. Hsu, S.H. et al. MicroRNA-122 Regulates Polyploidization in the Murine Liver. Hepatology (2016).

134. Margall-Ducos, G., Morizur-Celton, S., Couton, D., Bregerie, O. \& Desdouets, C. Liver Tetraploidization is Controlled by a New Process of Incomplete Cytokinesis. Journal of Cell Science 120, 3633-3639 (2007).

135. Wheatley, D.N. Binucleation in mammalian liver. Studies on the control of cytokinesis in vivo. Exp Cell Res 74, 455-65. (1972).

136. Girard, J., Ferre, P., Pegorier, J.P. \& Duee, P.H. Adaptations of glucose and fatty acid metabolism during perinatal period and suckling-weaning transition. Physiol Rev 72, 507-62 (1992).

137. Celton-Morizur, S., Merlen, G., Couton, D. \& Desdouets, C. Polyploidy and liver proliferation: central role of insulin signaling. Cell Cycle 9, 460-6 (2010).

138. Celton-Morizur, S., Merlen, G., Couton, D., Margall-Ducos, G. \& Desdouets, C. The insulin/Akt pathway controls a specific cell division program that leads to generation of binucleated tetraploid liver cells in rodents. J Clin Invest 119, 18807 (2009).

139. Wullschleger, S., Loewith, R. \& Hall, M.N. TOR signaling in growth and metabolism. Cell 124, 471-84 (2006).

140. Wong, J.V., Dong, P., Nevins, J.R., Mathey-Prevot, B. \& You, L. Network calisthenics: control of E2F dynamics in cell cycle entry. Cell Cycle 10, 3086-94 (2011).

141. Wilkinson, P.D. et al. The polyploid state restricts hepatocyte proliferation and liver regeneration. Hepatology (2018).

142. del Pozo, J.C., Diaz-Trivino, S., Cisneros, N. \& Gutierrez, C. The balance between cell division and endoreplication depends on E2FC-DPB, transcription factors regulated by the ubiquitin-SCFSKP2A pathway in Arabidopsis. Plant Cell 18, 2224-35 (2006).

143. Shibutani, S.T. et al. Intrinsic negative cell cycle regulation provided by PIP boxand Cul4Cdt2-mediated destruction of E2f1 during S phase. Dev Cell 15, 890-900 (2008).

144. Zielke, N. et al. Control of Drosophila endocycles by E2F and CRL4(CDT2). Nature 480, 123-7 (2011).

145. Qi, Q.R. et al. Involvement of atypical transcription factor E2F8 in the polyploidization during mouse and human decidualization. Cell Cycle 14, 1842-58 (2015).

146. Diril, M.K. et al. Cyclin-dependent kinase 1 (Cdk1) is essential for cell division and suppression of DNA re-replication but not for liver regeneration. Proc Natl Acad Sci U S A 109, 3826-31 (2012). 
147. Kurinna, S. et al. p53 regulates a mitotic transcription program and determines ploidy in normal mouse liver. Hepatology 57, 2004-13 (2013).

148. Sheahan, S., Bellamy, C.O., Treanor, L., Harrison, D.J. \& Prost, S. Additive effect of p53, p21 and Rb deletion in triple knockout primary hepatocytes. Oncogene 23, 1489-97 (2004).

149. Wu, H. et al. Targeted in vivo expression of the cyclin dependent kinase inhibitor p21 halts hepatocyte cell cycle progression, postnatal liver development and regeneration. Genes and Dev. 10, 245-260 (1996).

150. Baena, E. et al. c-Myc regulates cell size and ploidy but is not essential for postnatal proliferation in liver. Proc Natl Acad Sci U S A 102, 7286-91 (2005).

151. Nevzorova, Y.A. et al. Aberrant cell cycle progression and endoreplication in regenerating livers of mice that lack a single E-type cyclin. Gastroenterology 137, 691-703, 703 e1-6 (2009).

152. Li, D. et al. Hepatic loss of survivin impairs postnatal liver development and promotes expansion of hepatic progenitor cells in mice. Hepatology 58, 2109-21 (2013).

153. Kim, S.H. et al. Hepatocyte homeostasis for chromosome ploidization and liver function is regulated by Ssu72 protein phosphatase. Hepatology 63, 247-59 (2016).

154. Zhang, S. et al. Hippo Signaling Suppresses Cell Ploidy and Tumorigenesis through Skp2. Cancer Cell 31, 669-684 e7 (2017).

155. De Santis Puzzonia, M. et al. TGFbeta Induces Binucleation/Polyploidization in Hepatocytes through a Src-Dependent Cytokinesis Failure. PLoS One 11, e0167158 (2016).

156. Mayhew, C.N. et al. Liver-specific pRB loss results in ectopic cell cycle entry and aberrant ploidy. Cancer Res 65, 4568-77 (2005).

157. Magami, Y. et al. Cell proliferation and renewal of normal hepatocytes and bile duct cells in adult mouse liver. Liver 22, 419-25 (2002).

158. Geschwind, II, Alfert, M. \& Schooley, C. Liver regeneration and hepatic polyploidy in the hypophysectomized rat. Exp Cell Res 15, 232-5 (1958).

159. Faktor, V.M. \& Uryvaeva, I.V. [Progressive polyploidy in mouse liver following repeated hepatectomy]. Tsitologiia 17, 909-16 (1975).

160. Gerlyng, P. et al. Binucleation and polyploidization patterns in developmental and regenerative rat liver growth. Cell Prolif 26, 557-65 (1993).

161. Sigal, S.H. et al. Partial hepatectomy-induced polyploidy attenuates hepatocyte replication and activates cell aging events. Am J Physiol 276, G1260-72 (1999).

162. Maillet, V. et al. LKB1 as a Gatekeeper of Hepatocyte Proliferation and Genomic Integrity during Liver Regeneration. Cell Rep 22, 1994-2005 (2018).

163. Madra, S., Styles, J. \& Smith, A.G. Perturbation of hepatocyte nuclear populations induced by iron and polychlorinated biphenyls in C57BL/10ScSn mice during carcinogenesis. Carcinogenesis 16, 719-27 (1995).

164. Muramatsu, Y. et al. Increased polyploid incidence is associated with abnormal copper accumulation in the liver of LEC mutant rat. Res Commun Mol Pathol Pharmacol 107, 129-36 (2000).

165. Yamada, T. et al. Increased polyploidy, delayed mitosis and reduced protein phosphatase-1 activity associated with excess copper in the Long Evans Cinnamon rat. Res Commun Mol Pathol Pharmacol 99, 283-304 (1998). 
166. Lazzerini Denchi, E., Celli, G. \& de Lange, T. Hepatocytes with extensive telomere deprotection and fusion remain viable and regenerate liver mass through endoreduplication. Genes Dev 20, 2648-53 (2006).

167. Ahodantin, J. et al. Hepatitis B virus X protein promotes DNA damage propagation through disruption of liver polyploidization and enhances hepatocellular carcinoma initiation. Oncogene 38, 2645-2657 (2019).

168. Gentric, G. \& Desdouets, C. Liver polyploidy: Dr Jekyll or Mr Hide? Oncotarget 6, 8430-1 (2015).

169. Schmucker, D.L. Do hepatocytes age? Exp Gerontol 25, 403-12 (1990).

170. Schmucker, D.L. Hepatocyte fine structure during maturation and senescence. $J$ Electron Microsc Tech 14, 106-25 (1990).

171. Asahina, K. et al. Multiplicative mononuclear small hepatocytes in adult rat liver: their isolation as a homogeneous population and localization to periportal zone. Biochem Biophys Res Commun 342, 1160-7 (2006).

172. Knouse, K.A., Lopez, K.E., Bachofner, M. \& Amon, A. Chromosome Segregation Fidelity in Epithelia Requires Tissue Architecture. Cell 175, 200-211 e13 (2018).

173. Overturf, K., Al-Dhalimy, M., Finegold, M. \& Grompe, M. The repopulation potential of hepatocyte populations differing in size and prior mitotic expansion. Am J Pathol 155, 2135-43 (1999).

174. Ganem, N.J. et al. Cytokinesis failure triggers hippo tumor suppressor pathway activation. Cell 158, 833-48 (2014).

175. Wang, M.J., Chen, F., Lau, J.T.Y. \& Hu, Y.P. Hepatocyte polyploidization and its association with pathophysiological processes. Cell Death Dis 8, e2805 (2017).

176. Wang, M.J. et al. Reversal of hepatocyte senescence after continuous in vivo cell proliferation. Hepatology 60, 349-61 (2014).

177. Schoenfelder, K.P. \& Fox, D.T. The expanding implications of polyploidy. J Cell Biol 209, 485-91 (2015).

178. Rios, A.C. et al. Essential role for a novel population of binucleated mammary epithelial cells in lactation. Nat Commun 7, 11400 (2016).

179. Tanami, S. et al. Dynamic zonation of liver polyploidy. Cell Tissue Res 368, 405410 (2017).

180. Coate, J.E. \& Doyle, J.J. Quantifying whole transcriptome size, a prerequisite for understanding transcriptome evolution across species: an example from a plant allopolyploid. Genome Biol Evol 2, 534-46 (2010).

181. Miettinen, T.P. et al. Identification of transcriptional and metabolic programs related to mammalian cell size. Curr Biol 24, 598-608 (2014).

182. Lu, P. et al. Microarray analysis of gene expression of mouse hepatocytes of different ploidy. Mamm Genome 18, 617-26 (2007).

183. Kreutz, C. et al. Hepatocyte Ploidy Is a Diversity Factor for Liver Homeostasis. Front Physiol 8, 862 (2017).

184. Anatskaya, O.V. \& Vinogradov, A.E. Genome multiplication as adaptation to tissue survival: evidence from gene expression in mammalian heart and liver. Genomics 89, 70-80 (2007).

185. Anatskaya, 0.V. \& Vinogradov, A.E. Somatic polyploidy promotes cell function under stress and energy depletion: evidence from tissue-specific mammal transcriptome. Funct Integr Genomics 10, 433-46 (2010).

186. Bahar Halpern, K. et al. Bursty gene expression in the intact mammalian liver. Mol Cell 58, 147-56 (2015). 
187. Eldar, A. \& Elowitz, M.B. Functional roles for noise in genetic circuits. Nature 467, 167-73 (2010).

188. Knouse, K.A., Wu, J., Whittaker, C.A. \& Amon, A. Single cell sequencing reveals low levels of aneuploidy across mammalian tissues. Proc Natl Acad Sci U S A 111, 13409-14 (2014).

189. McConnell, M.J. et al. Mosaic copy number variation in human neurons. Science 342, 632-7 (2013).

190. Weier, J.F. et al. Human cytotrophoblasts acquire aneuploidies as they differentiate to an invasive phenotype. Dev Biol 279, 420-32 (2005).

191. Westra, J.W. et al. Aneuploid mosaicism in the developing and adult cerebellar cortex. J Comp Neurol 507, 1944-51 (2008).

192. Duncan, A.W. et al. Aneuploidy as a mechanism for stress-induced liver adaptation. J Clin Invest 122, 3307-15 (2012).

193. Matsumoto, T., Wakefield, L., Tarlow, B.D. \& Grompe, M. In Vivo Lineage Tracing of Polyploid Hepatocytes Reveals Extensive Proliferation during Liver Regeneration. Cell Stem Cell (2019).

194. Grompe, M. \& al-Dhalimy, M. Rapid nonradioactive assay for the detection of the common French Canadian tyrosinemia type I mutation. Hum Mutat 5, 105 (1995).

195. Wilkinson, P.D. et al. Polyploid Hepatocytes Facilitate Adaptation and Regeneration to Chronic Liver Injury. Am J Pathol 189, 1241-1255 (2019).

196. Losick, V.P., Fox, D.T. \& Spradling, A.C. Polyploidization and cell fusion contribute to wound healing in the adult Drosophila epithelium. Curr Biol 23, 2224-2232 (2013).

197. Losick, V.P. Wound-Induced Polyploidy Is Required for Tissue Repair. Adv Wound Care (New Rochelle) 5, 271-278 (2016).

198. Zhang, S. et al. The Polyploid State Plays a Tumor-Suppressive Role in the Liver. Dev Cell 47, 390 (2018).

199. Sato, N. et al. Centrosome abnormalities in pancreatic ductal carcinoma. Clin Cancer Res 5, 963-70 (1999).

200. Lothschutz, D. et al. Polyploidization and centrosome hyperamplification in inflammatory bronchi. Inflamm Res 51, 416-22 (2002).

201. Hamada, S., Itoh, R. \& Fujita, S. DNA distribution pattern of the so-called severe dysplasias and small carcinomas of the colon and rectum and its possible significance in the tumor progression. Cancer 61, 1555-62 (1988).

202. Montgomery, B.T. et al. Stage B prostate adenocarcinoma. Flow cytometric nuclear DNA ploidy analysis. Arch Surg 125, 327-31 (1990).

203. Bielski, C.M. et al. Genome doubling shapes the evolution and prognosis of advanced cancers. Nat Genet 50, 1189-1195 (2018).

204. Dewhurst, S.M. et al. Tolerance of whole-genome doubling propagates chromosomal instability and accelerates cancer genome evolution. Cancer Discov 4, 175-185 (2014).

205. Galipeau, P.C. et al. 17p (p53) allelic losses, 4N (G2/tetraploid) populations, and progression to aneuploidy in Barrett's esophagus. Proc Natl Acad Sci U S A 93, 7081-4 (1996).

206. Maley, C.C. et al. Genetic clonal diversity predicts progression to esophageal adenocarcinoma. Nat Genet 38, 468-73 (2006).

207. Kneissig, M., Bernhard, S. \& Storchova, Z. Modelling chromosome structural and copy number changes to understand cancer genomes. Curr Opin Genet Dev 54, 25-32 (2019). 
208. Tanaka, K. et al. Tetraploidy in cancer and its possible link to aging. Cancer Sci 109, 2632-2640 (2018).

209. Bloy, N. et al. Immunogenic stress and death of cancer cells: Contribution of antigenicity vs adjuvanticity to immunosurveillance. Immunol Rev 280, 165-174 (2017).

210. Senovilla, L. et al. An immunosurveillance mechanism controls cancer cell ploidy. Science 337, 1678-84 (2012).

211. Acebes-Huerta, A. et al. Drug-induced hyperploidy stimulates an antitumor NK cell response mediated by NKG2D and DNAM-1 receptors. Oncoimmunology 5, e1074378 (2016).

212. Aranda, F. et al. Immune effectors responsible for the elimination of hyperploid cancer cells. Oncoimmunology 7, e1463947 (2018).

213. Cosenza, M.R. \& Kramer, A. Centrosome amplification, chromosomal instability and cancer: mechanistic, clinical and therapeutic issues. Chromosome Res 24, 105-26 (2016).

214. Ganem, N.J., Godinho, S.A. \& Pellman, D. A mechanism linking extra centrosomes to chromosomal instability. Nature 460, 278-82 (2009).

215. Wangsa, D. et al. Near-tetraploid cancer cells show chromosome instability triggered by replication stress and exhibit enhanced invasiveness. FASEB J 32, 3502-3517 (2018).

216. Burrell, R.A., McGranahan, N., Bartek, J. \& Swanton, C. The causes and consequences of genetic heterogeneity in cancer evolution. Nature 501, 338-45 (2013).

217. Lv, L. et al. Tetraploid cells from cytokinesis failure induce aneuploidy and spontaneous transformation of mouse ovarian surface epithelial cells. Cell Cycle 11, 2864-75 (2012).

218. Zhang, S., Lin, Y.H., Tarlow, B. \& Zhu, H. The origins and functions of hepatic polyploidy. Cell Cycle 18, 1302-1315 (2019).

219. Austin, L.S., Kaushansky, A. \& Kappe, S.H. Susceptibility to Plasmodium liver stage infection is altered by hepatocyte polyploidy. Cell Microbiol 16, 784-95 (2014). 


\section{Acknowledgements}

2 The authors are supported by French grants from the Institut National de la Santé et de la

3 Recherche Médicale (INSERM), la Fondation pour la Recherche Médicale (Equipe FRM:

4 EQU201903007824), the Institut National du Cancer (PRTK-2017, PLBIO18-107), the

5 Agence Nationale de Recherche ANR (ANR-16-CE14), Fondation ARC (Association de

6 Recherche sur le Cancer), Ligue Contre le Cancer (comité de Paris), the Cancéropôle Ile-de-

7 France (Emergence 2015), The Association Française pour l'Etude du Foie (AFEF-SUBV

8 2017), EVA-Plan Cancer INSERM HTE and the SIRIC CARPEM. R.D. is a recipient of

9 Fondation pour la Recherche Médicale ( $\mathrm{PhD}$ grant). P.C. is a recipient of Plan Cancer

10 INSERM (program «Soutien pour la formation à la recherche fondamentale et 11 translationnelle en cancérologie $»)$.

12 
1 Fig. 1 | Characteristics of polyploidy. a| Ploidy is the number of complete sets of

2 chromosomes in a cell. Somatic individual organisms, tissues and cells can be described

3 according to the number of sets of chromosomes present: haploid (1 set), diploid (2 sets),

4 tetraploid (4 sets). b | Polyploidy is defined on the basis of the DNA content of each nucleus,

5 which is called nuclear ploidy (for example, a cell can be tetraploid mononucleate $(4 n)$ or

6 octoploid mononucleate $(8 n)$ ), and on the number of nuclei per cell, which is called cellular 7 ploidy (for example, a cell can be tetraploid binucleate $(2 \times 2 n)$ or hexaploid trinucleate $8(3 \times 2 n)) . n$, chromosome number.

Fig. 2 | Molecular mechanisms that lead to the polyploid state. Polyploid cells can be generated through various mechanisms that are classified according to their dependence on the cell cycle. $\mathbf{a} \mid$ The only cell cycle-independent process that leads to polyploid cells is cell 13 fusion, which can occur during virus-mediated infections or through receptor-ligand 14 interactions, giving rise to binucleate or multinucleated cells. $\mathbf{b} \mid$ The other mechanism relies on modifications of the canonical eukaryotic cell cycle that arise either during interphase (G1, S and G2) or mitosis (prophase, metaphase, anaphase and telophase). c | Endoreplication encompasses both endocycling and endomitosis. (1) Endocycling is comprised of alternating $\mathrm{G}$ and $\mathrm{S}$ phases, which eventually results in a rise in nuclear ploidy. This aberrant cell cycle is achieved by the combination of S-phase cyclin-dependent kinase (S-CDK) oscillation and mitotic entry impediment, either through cyclin B proteolysis or M-phase cyclin-dependent kinase (M-CDK) inhibition. (2) Endomitosis stems from issues that arise during mitosis. For instance, prolonged spindle assembly checkpoint (SAC) activation in metaphase resulting from a non-amphitelic chromosome attachment can be accompanied by progressive cyclin B1

24 degradation. This process, known as mitotic slippage, allows the cell to escape mitosis and 25 reach G1. (3) Finally, cytokinesis failure can result in the genesis of binucleate cells by 
1 preventing the physical separation of the cytoplasm (through cytokinesis) in telophase.

2 Cytokinesis failure can be induced as a result of any interferences emerging during the highly

3 regulated process of cytokinesis. Lightning bolts indicate the location of cell-cycle anomalies,

4 such as lagging chromosomes or cytoskeleton disorganization.

\section{$5 \quad$ Fig. 3 | Polyploidization during post-natal liver growth.}

6 a $\mid$ Polyploidization of hepatic tissue is a progressive developmental process that takes place

7 around the time of weaning and is regulated by insulin, PI3K-mTORC2, E2F and miR-122

8 signalling. At birth, hepatocytes are exclusively diploid mononucleate (2n). During post-natal

9 liver development after weaning, diploid hepatocytes can either enter the normal cell cycle

10 (indicated by the blue arrow), giving rise to two diploid hepatocytes, or follow an aberrant

11 cell cycle that is characterized by incomplete cytokinesis (indicated by the orange arrow),

12 giving rise to one binucleate tetraploid $(2 \times 2 n)$ hepatocyte. Progressive polyploidization takes

13 place via this process in the liver parenchyma, and tetraploid and octoploid cells with one or

14 two nuclei are formed. Up to $90 \%$ of adult hepatocytes in rodents and approximately $30 \%$ in

15 humans are polyploid. b| During the complete cytokinesis process (left panel), the cytokinetic

16 machinery (MgcRacGAP; red; left upper panel) localized during anaphase both on the central

17 spindle and on astral equatorial microtubules and during telophase at the midbody.

18 Consequently, RhoA (green; left lower panel), the major cytokinesis orchestrator,

19 accumulates at the equatorial cortex in early telophase, leading to the formation of the

20 cytokinetic ring and the genesis of two diploid progenies. During incomplete cytokinesis

21 (right panel), microtubules are disorganized (green; right upper panel). Consequently,

22 MgcRacGAP (red; right upper panel) is observed on the remaining interdigitating

23 microtubules in anaphase and telophase but is never localized on unattached astral equatorial

24 microtubules, indicating that molecular signals delivered by microtubules to the equatorial

25 cortex are impaired. Furthermore, RhoA (green; right lower panel) does not correctly localize 
1 at the equatorial cortex and is instead observed near the cell centre, close to central spindle

2 microtubules. Consequently, activation of RhoA GTPase in the central cortex is impaired,

3 leading to the genesis of binucleate progeny. Adapted from Figure $6^{134}$.

4

5 Fig. 4 | The genesis of mononucleate polyploid hepatocytes in physiopathological

contexts. In human and mouse models of nonalcoholic fatty liver disease, the oxidative stress caused by an increase in levels of reactive oxygen species (ROS) induces the activation of the DNA damage response (ATR-p53-p21). This activation leads to multiple endoreplication

9 cycles, generating polyploid mononucleate hepatocytes (for example, a tetraploid mononucleate cell $(4 n)$ or an octoploid mononucleate cell $(8 n))$. During liver regeneration, there is also a shift from cellular ploidy to nuclear ploidy (for example, a diploid binucleate cell can form two tetraploid mononucleate cells, and a tetraploid binucleate cell can form two octoploid mononucleate cells or a $16 n$ mononucleate cell). The hepatic parenchyma is also enriched in polyploid mononucleate hepatocytes (for example, $4 n$ and $8 n$ cells) following an iron or copper overload. $n$, chromosome number.

Fig. 5 | The polyploid state: gatekeeper or driver of liver tumorigenesis? Depending on the context and especially on the TP53 status (that is, mutated or wild-type), the polyploid state can be regarded as a gatekeeper or a driver of hepatocarcinogenesis. Indeed, polyploid hepatocytes (for example, $4 n$ and $8 n$ ) are more resistant to oncogenic events than diploid

21 hepatocytes because they possess extra copies of tumour suppressor genes, which confers 22 protection against loss of heterozygosity by buffering the effects of inactivating mutations. 23 Furthermore, the polyploid state can hinder hepatocyte proliferation by means of the p5324 dependent 'tetraploid checkpoint', which restricts proliferation and favours commitment to 
1 cell death. It is therefore thought that liver tumours stem from diploid hepatocytes. TP53

2 mutation represents a crucial event in the development of polyploid tumors, which are

3 associated with enhanced aggressiveness and poor prognosis. Indeed, interfering with the p53

4 pathway allows polyploid cells to resume the cell cycle despite exhibiting deleterious

5 chromosomal instability, which ultimately leads to aneuploidy. Hence, the polyploid state acts

6 as a tumour-promoting mechanism by amplifying genomic aberrations and exacerbating

7 tumour aggressiveness. However, it remains unknown whether polyploid tumours arise from

8 a proliferating polyploid clone or from an initiating diploid tumour that has subsequently

9 undergone polyploidization.

10

11

12

13

14 


\section{Figure 1:}

HAPLOID STATE

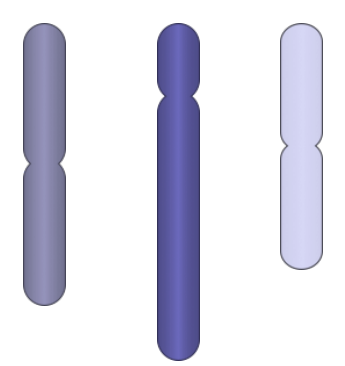

DIPLOID STATE

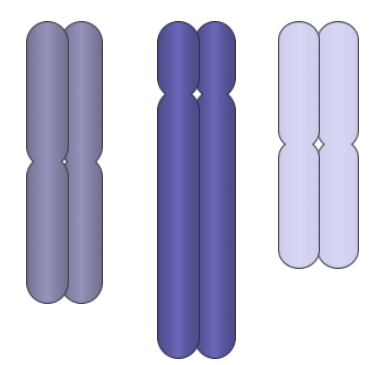

TETRAPLOID STATE

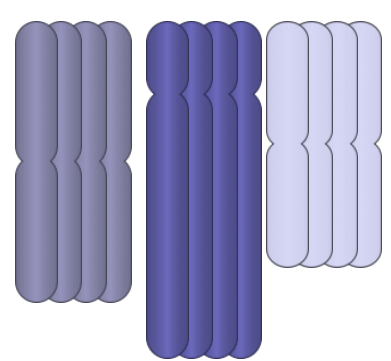

Nuclear ploidy

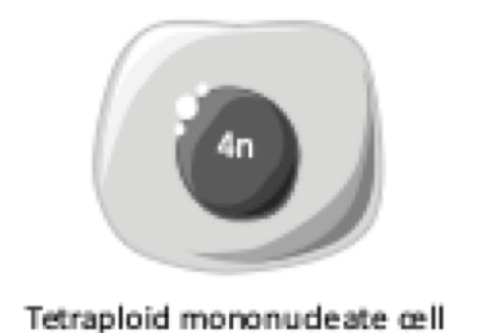

Tetraploid mononudeate well

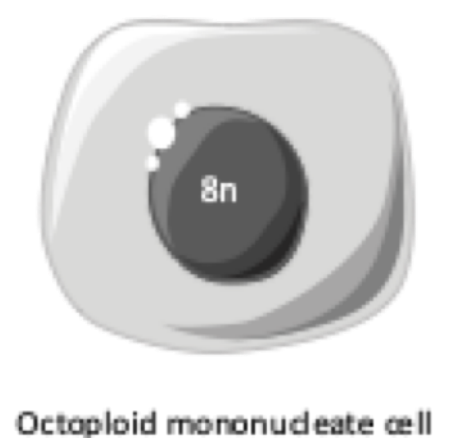

Octoploid mononudeate cell

\section{Cellular ploidy}

\section{$2 n+2 n$}

Tetraploid binucleate cell

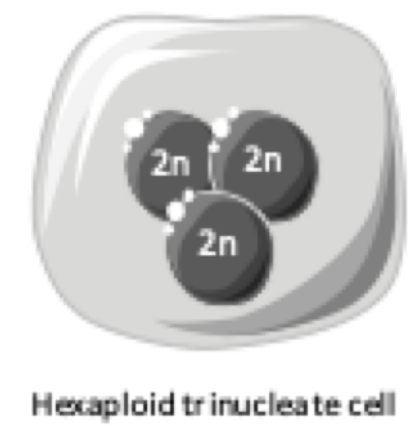




\section{Figure 2:}

A

INDEPENDENT OF CELL CYCLE (Cell fusion)

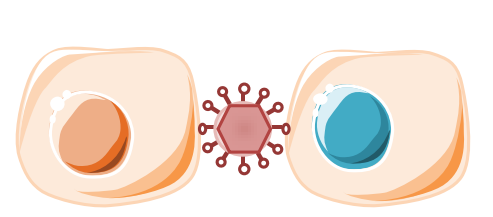

Virusdependent $\longrightarrow$

Receptor-

ligand

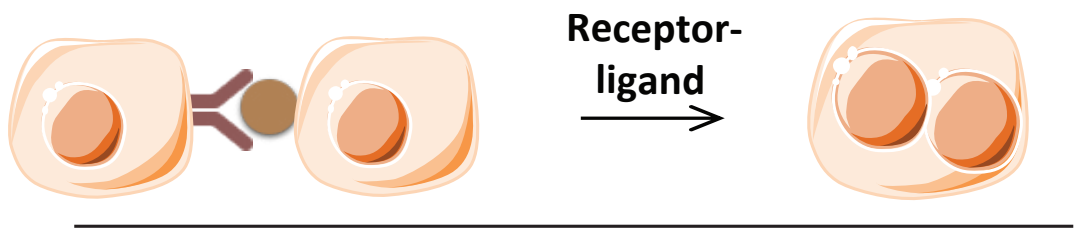

B DEPENDENT OF CELL CYCLE

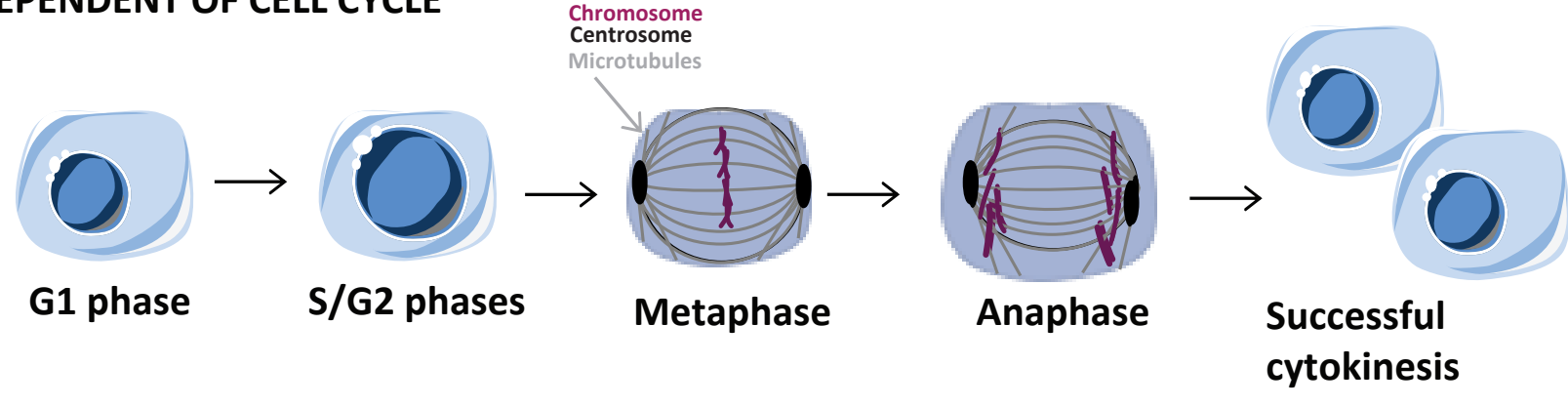




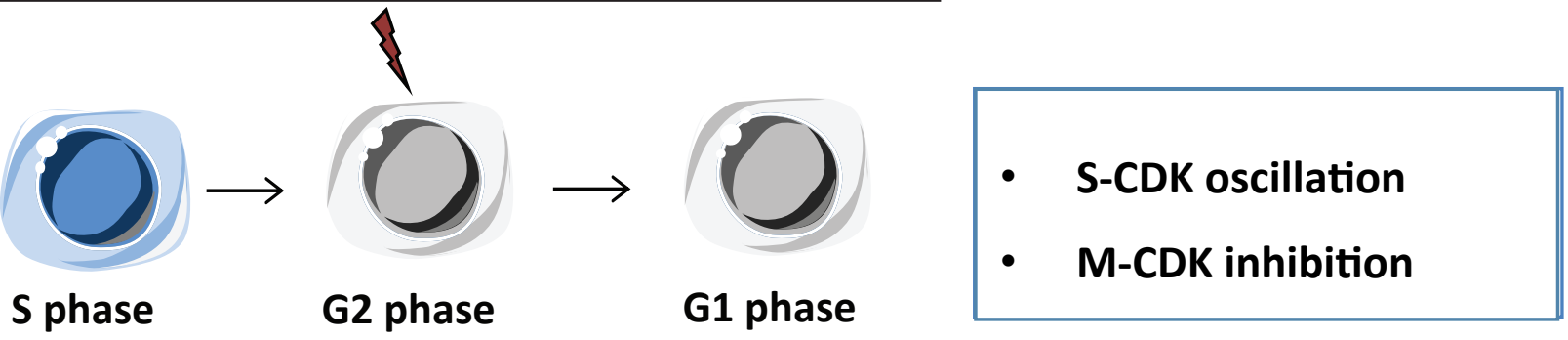

2 Endoreplication / Endomitosis

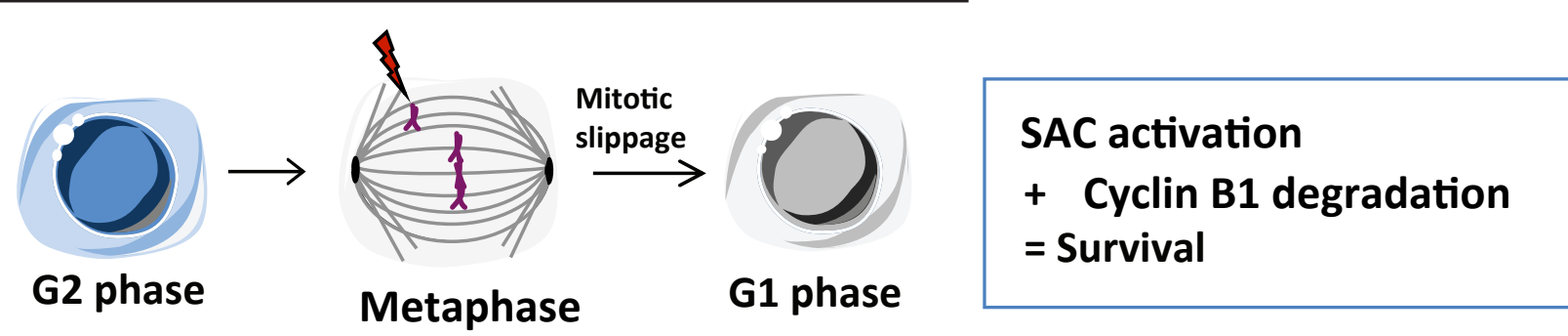

3 Cytokinesis failure

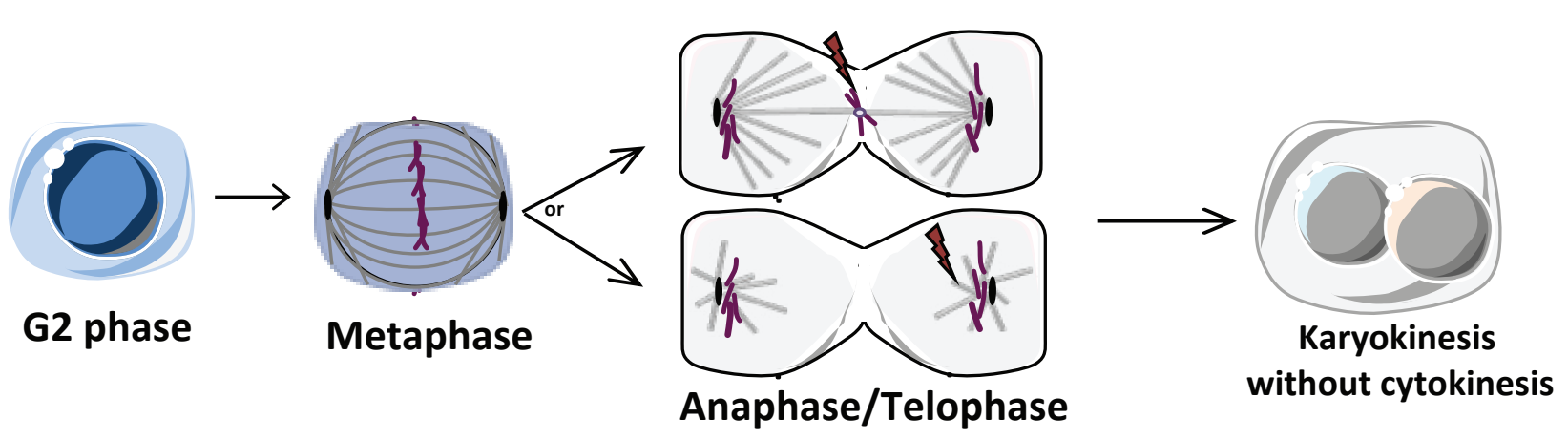


Figure 3:

A

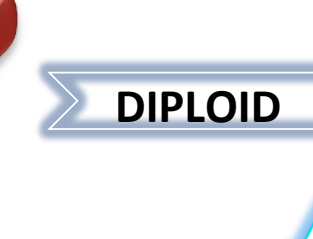

POLYPLOID

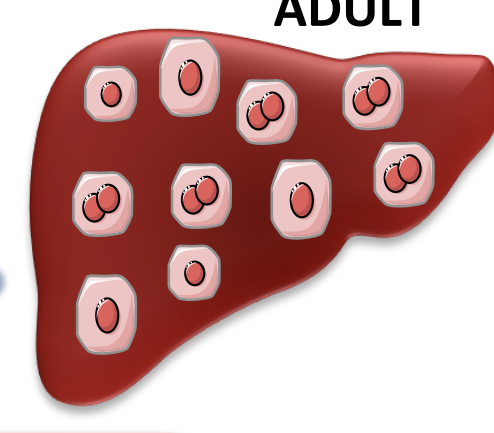

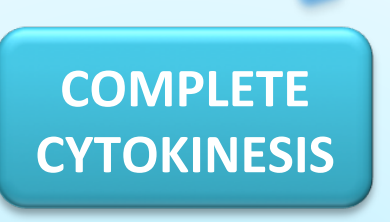

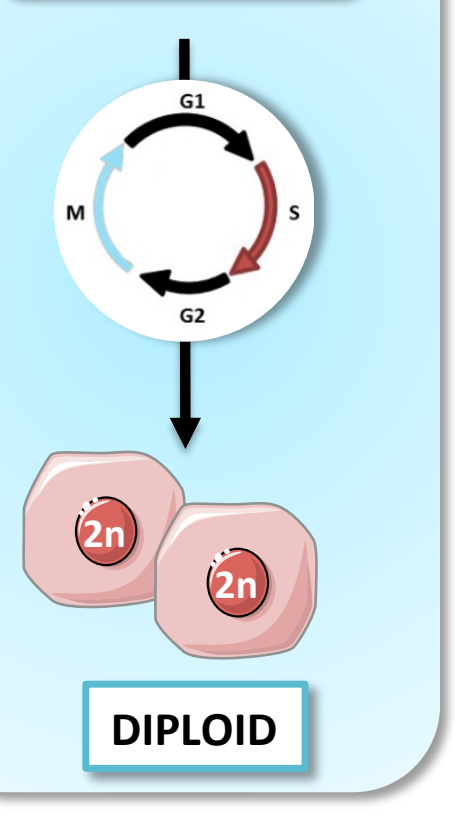

\section{INCO}

INCOMPLETE

CYTOKINESIS

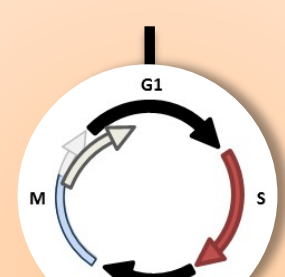

1

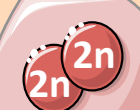

TETRAPLOID

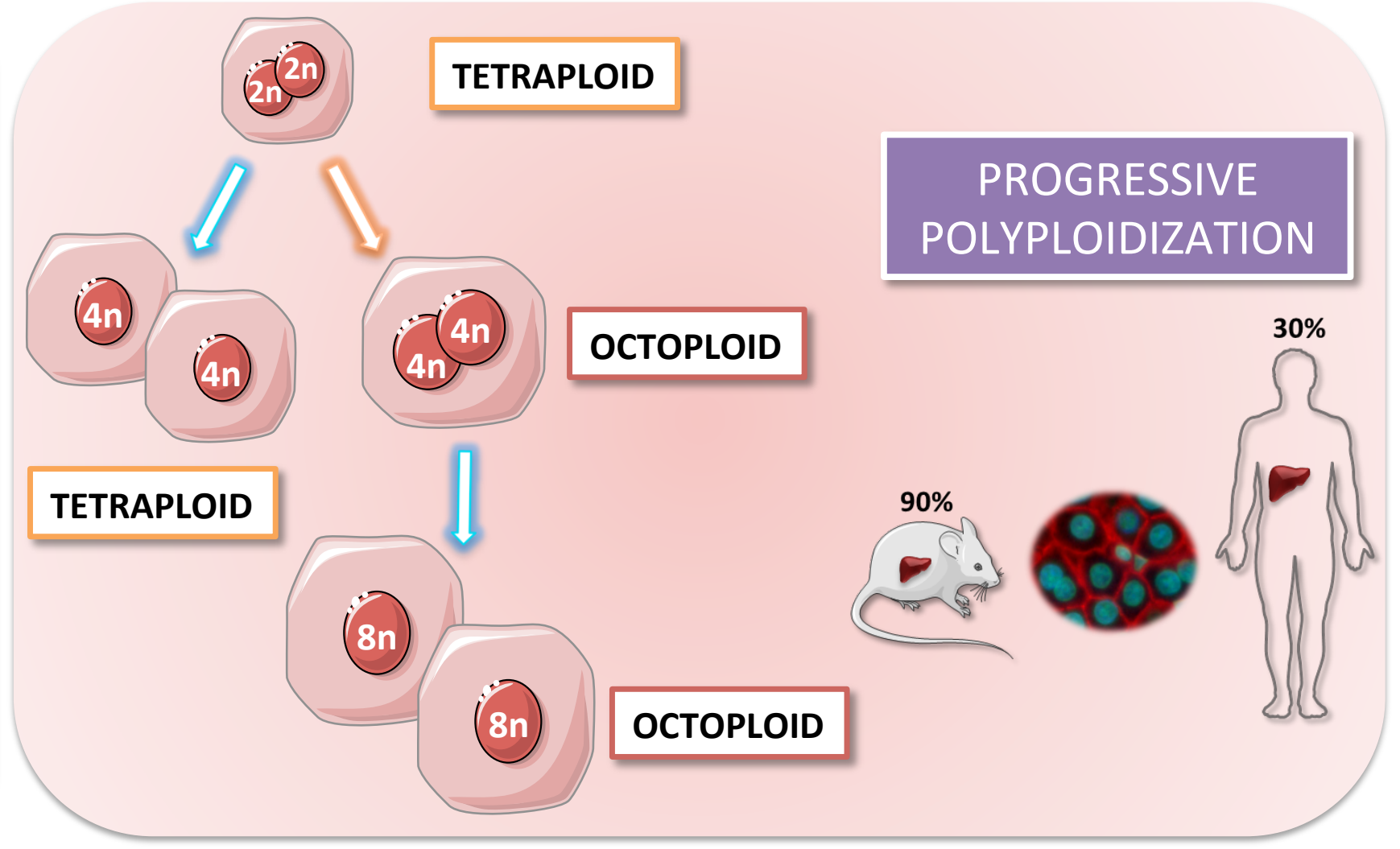


Figure 3:

B

COMPLETE CYTOKINESIS

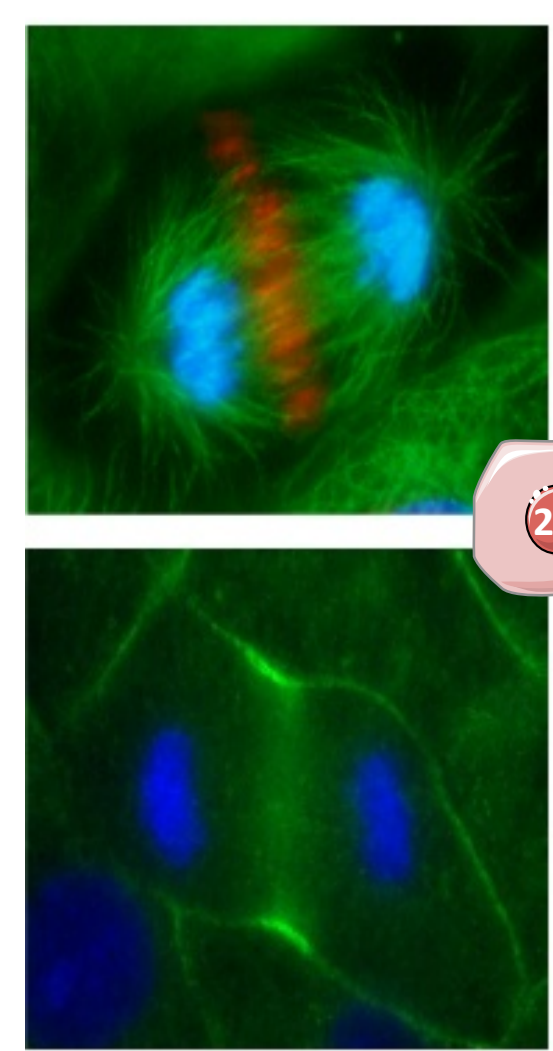

Anaphase

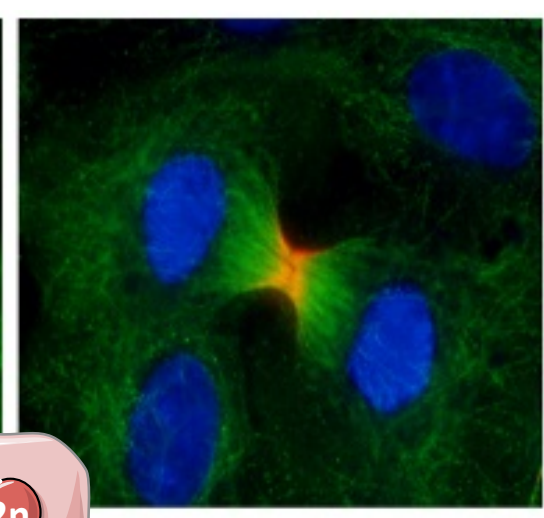

(2in)

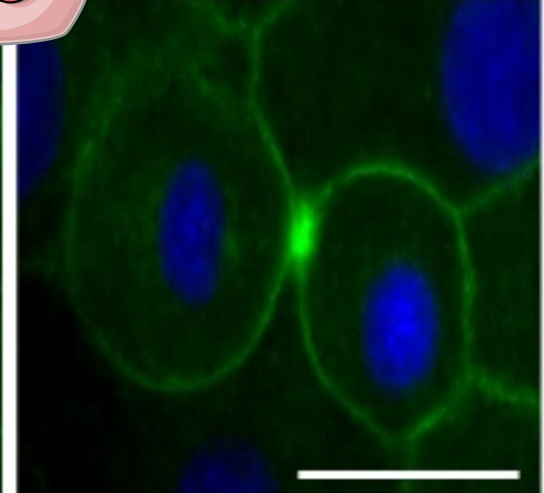

Telophase
INCOMPLETE CYTOKINESIS

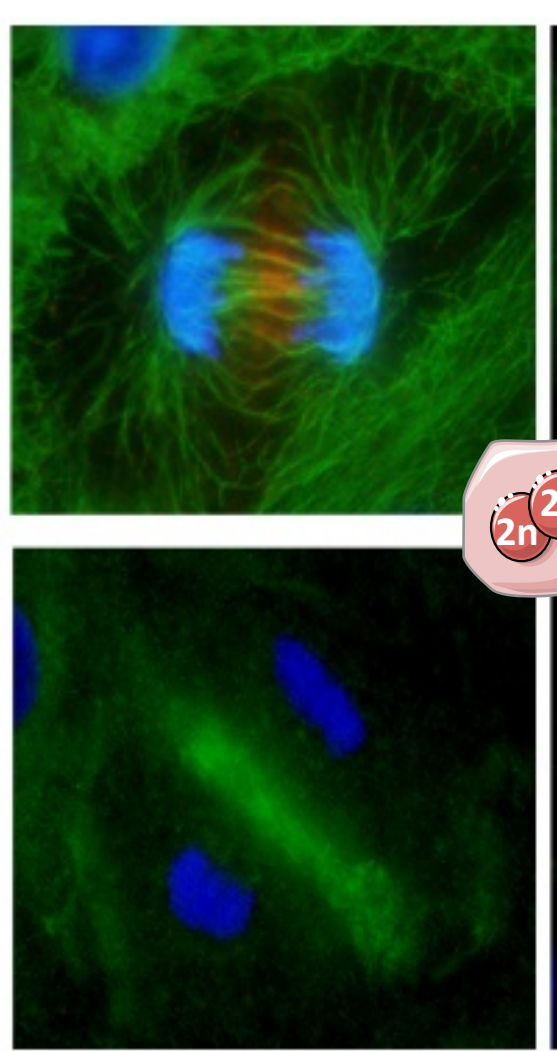

Anaphase
Hoechst

$\beta$-tubulin

- MgcRacGAP

Hoechst

RhoA 


\section{Figure 4:}
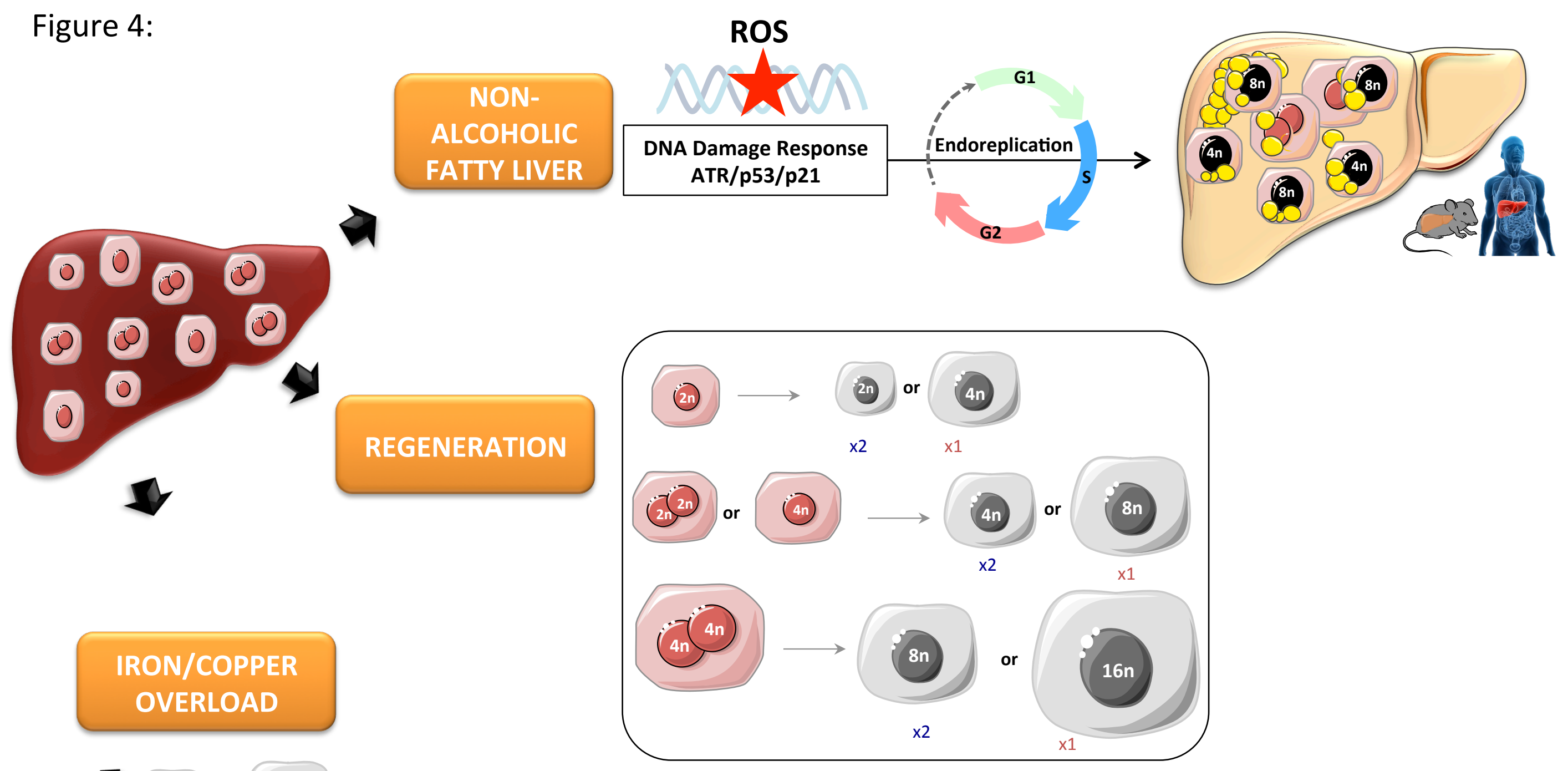

10 
Figure 5:

\section{ONCOGENIC EVENTS}

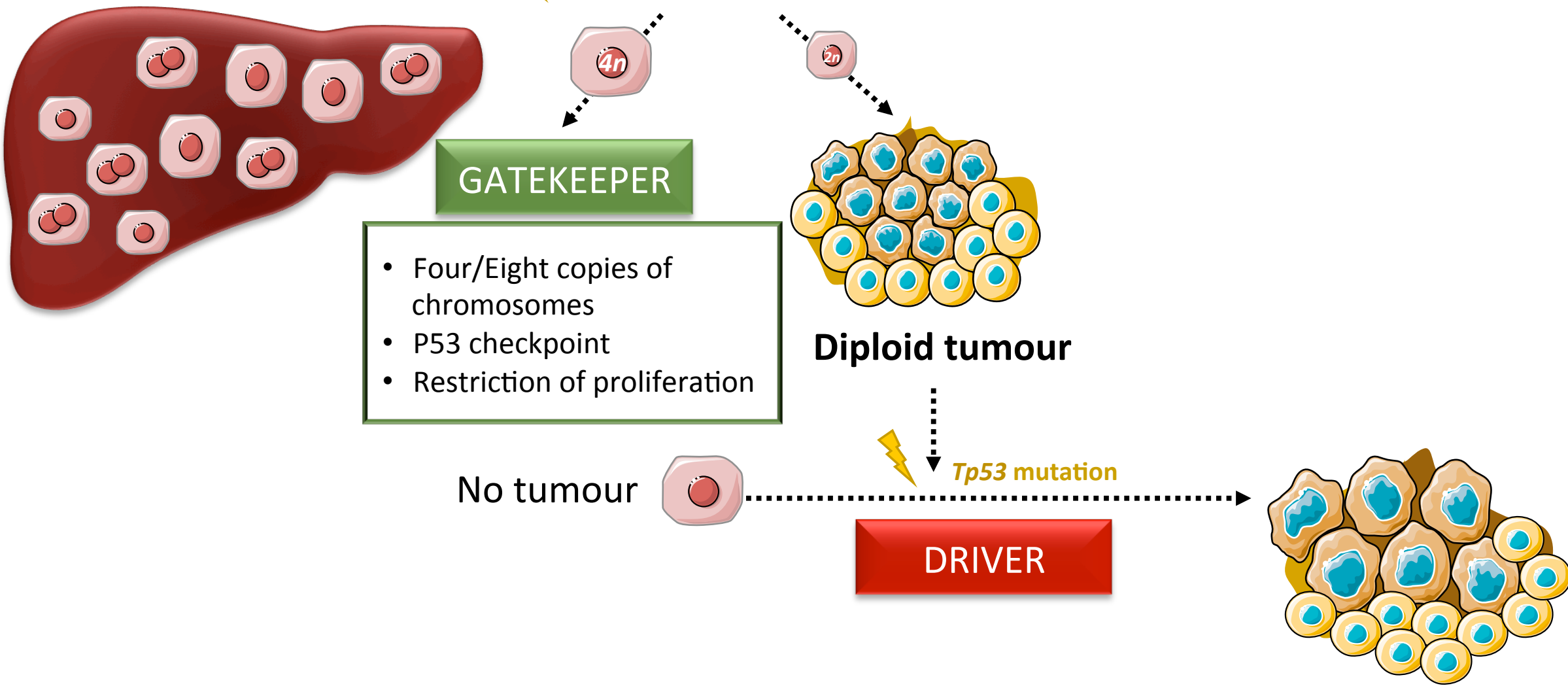

Polyploid tumour

- HIGHER AGGRESSIVENESS

Proliferation rate

Differentiation

- POORER PROGNOSIS

$\nearrow$ Disease relapse 